\title{
Estimación de edad, crecimiento y mortalidad natural de la merluza de cola, Macruronus magellanicus Lönnberg, 1907 (Macruronidae, Gadiformes) en el Océano Pacífico Suroriental
}

\author{
Determination of age, growth and natural mortality of Chilean hoki, Macruronus magellanicus, \\ Lönnberg, 1907 (Macruronidae, Gadiformes) from the Southeastern Pacific Ocean \\ Javier V. Chong ${ }^{1}$, Mario Aguayo ${ }^{1}$ e Ignacio Payá ${ }^{2}$ \\ ${ }^{1}$ Universidad Católica de la Santísima Concepción, Casilla 297, Concepción, Chile \\ ${ }^{2}$ Instituto de Fomento Pesquero, Casilla 8-V, Valparaíso, Chile \\ jvchong@ucsc.cl
}

\begin{abstract}
Age, growth and natural mortality in Chilean hoki, Macruronus magellanicus, are estimated using samples of saggital otoliths from specimens caught from 1991 to 1996 between $46^{\circ}$ and $56^{\circ} \mathrm{S}$. The age determination was done by reading of annual growth ring in otoliths. The lengths at different ages were obtained by back calculation and also to actual ages. The validation of the annual formation of otoliths increment was established by the regularity of the deposition of the hyaline and opaque rings in the edge of the otoliths and the marginal increment analysis. This allowed the determination of the formation of an annual hyaline (annulus) in July and an opaque ring in January. The growth was determined following the von Bertalanffy model and the growth parameters were assessed separately for each sex, using a nonlinear adjustment model. The growth parameters between sexes were compared with the $\mathrm{T}^{2}$ test of Hotelling and the minimum square ratio of Chen et al. (1992). The results indicate that growth was significantly greater in females than in males. The results obtained here are consistent with previous records for the same species from other regions of the Eastern Pacific, the Western Atlantic, as well as with the congeneric species $M$. novaezelandiae, from the coasts of Australia and New Zealand. The instantaneous coefficient of natural mortality (M) was estimated with empirical methods and catch-curve analysis.
\end{abstract}

Key words: Macruronus, annuli, growth, mortality
Resumen.- Se presenta un estudio de edad, crecimiento y mortalidad natural en merluza de cola, Macruronus magellanicus, realizado con muestras de otolitos saculares de especímenes capturados en el periodo 1991- 1996 entre las latitudes $46^{\circ} \mathrm{S}$ y $56^{\circ} \mathrm{S}$. La determinación de edad se efectuó por el método de lectura de anillos anuales de crecimiento presentes en los otolitos. Las longitudes a las distintas edades se estimaron mediante un método de retrocálculo de longitudes (a edades pretéritas), así como también a edad actual. La validación de la periodicidad de la depositación de los anillos hialinos y opacos se estableció de acuerdo al análisis mensual del tipo de borde en los otolitos, determinándose la formación de un anillo hialino anual (annulus), en julio y un anillo opaco en enero. El crecimiento se determinó utilizando el modelo de von Bertalanffy y los parámetros de crecimiento se estimaron en forma independiente por sexo, empleándose un modelo de ajuste no lineal. La comparación de los parámetros de crecimiento entre los sexos se realizó mediante la prueba $\mathrm{T}^{2}$ de Hotelling y la razón de mínimos cuadrados de Chen et al. (1992). Los resultados señalan que el crecimiento fue significativamente mayor en las hembras que en los machos. Los resultados obtenidos concuerdan en gran medida con lo registrado para la misma especie en otras áreas del O. Pacífico Este, del O. Atlántico, y también en la especie congenérica $M$. novaezelandiae de las costas de Australia y Nueva Zelanda. El coeficiente instantáneo de mortalidad natural (M) fue estimado mediante métodos bioanalógicos y análisis de curva de captura.

Palabras clave: Macruronus, annuli, crecimiento, mortalidad

\section{Introducción}

Macruronus magellanicus Lönnberg, 1907 conocida en Chile con el nombre vernacular de merluza de cola, colihuasca y huaica, e internacionalmente como "Chilean hoki" se distribuye en el cono sur de
Sudamérica, desde la latitud $37^{\circ} \mathrm{S}$ en el Océano Atlántico, hasta los $27^{\circ} 03^{\prime} \mathrm{S}$ en el Océano Pacífico, en profundidades de 20 a $800 \mathrm{~m}$ en la costa argentina (Angelescu et al. 1958, Chesheva 1992), y desde los 20 a $700 \mathrm{~m}$ en la costa chilena (Arana 1970, Avilés et al. 1979, Cohen et al. 1990). Adicionalmente, en el litoral 
de Chile, durante los últimos años se han registrado capturas frente al puerto de Caldera (27\%03'S) (SERNAPESCA 1999, 2000).

La merluza de cola es objeto de una pesquería de arrastre y de cerco industrial, tanto en la plataforma argentina como frente a la costa de Chile. Las capturas en el litoral chileno se iniciaron en 1978 como fauna acompañante de la merluza del sur (Merluccius australis Hutton) en la zona de la pesquería sur-austral, (4300’- 5700’S) (Aguayo et al. 1986, 1987, 1991). Estas capturas muestran una tendencia creciente desde los inicios de la pesquería en el año 1978, con desembarques de aproximadamente $14.000 \mathrm{t}$ efectuada por la flota arrastrera en la zona sur-austral, para alcanzar el máximo de 375.000 t en 1996, cantidad que incluye el desembarque de la flota cerquera en la zona

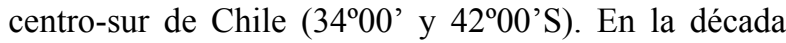
1991-2000, las capturas de merluza de cola han alcanzado un total de $1.988 .660 \mathrm{t}$, con el máximo señalado anteriormente en 1996, siendo 1.824.104 t capturadas mediante arte de cerco entre las latitudes $34^{\circ} 00^{\prime} \mathrm{S}$ y $42^{\circ} 00^{\prime} \mathrm{S}$, las que tienen como destino la elaboración de harina de pescado (SERNAPESCA 2000). Los restantes volúmenes de captura son extraídos en la zona sur-austral por embarcaciones que usan redes de arrastre y media agua, siendo en los últimos años destinada en gran parte a productos congelados para exportación.

La pesquería en Chile se localiza en la zona centrosur donde opera principalmente una flota industrial de cerco al sur de Isla Mocha, con un límite oeste tentativo en $74^{\circ} 30^{\prime} \mathrm{W}$ y un límite sur en la latitud $40^{\circ} 30^{\prime} \mathrm{S}$ (Cubillos et al. 1998). En la zona sur austral opera una flota de arrastre de fondo y media agua en zonas de pesca que se localizan en aguas exteriores del sistema de fiordos y canales, principalmente desde los $43^{\circ} 00^{\prime} \mathrm{S}$ hasta los $57^{\circ} 25^{\prime} \mathrm{S}$. En esta pesquería se identifican tres zonas o caladeros, denominadas Norte $\left(43^{\circ} 00^{\prime}-\right.$ $\left.50^{\circ} 00^{\prime} \mathrm{S}\right)$, Centro $\left(50^{\circ} 00^{\prime}-55^{\circ} 25^{\prime} \mathrm{S}\right)$ y Sur entre los $55^{\circ} 25^{\prime}$ y $57^{\circ} 25^{\prime} \mathrm{S}$ (Young et al. 1998).

La mayor biomasa total estimada mediante la aplicación de un modelo analítico fue de alrededor de cuatro millones de toneladas en 1995 (Payá et al. 2003, 2005), experimentando una disminución sostenida en los años siguientes hasta aproximadamente $700 \mathrm{mil}$ toneladas, el 2004. Esta disminución ha estado asociada con una fuerte disminución del stock desovante y de la fracción juvenil (reclutas).
La estructura de talla de los desembarques indica que en la pesquería sur-austral las capturas comprenden desde los 30 a los $118 \mathrm{~cm}$ de longitud total (LT), donde sobre el $80 \%$ lo constituyen ejemplares adultos, superiores a los $57 \mathrm{~cm}$ LT que corresponde a la talla de primera madurez sexual. Mientras que, en la zona centro-sur, las tallas capturadas están entre 29 y $79 \mathrm{~cm}$ LT, pero donde un porcentaje superior al $80 \%$ de las capturas corresponden a ejemplares inferiores a $57 \mathrm{~cm}$ de LT (Arancibia et al. 1994), lo que implica que la pesquería se realiza sobre la fracción juvenil de la población en esta área de pesca. No obstante, el aumento sostenido de las capturas no se ha traducido en un aumento proporcional sobre su conocimiento biológico. En particular, hay escasos estudios de la biología reproductiva, de la determinación de una función representativa del crecimiento, reconocimiento de unidad de stock y áreas de desove, entre otros aspectos.

Los antecedentes biológicos más relevantes de esta especie son aquellos relacionados con su crecimiento, los cuales se han efectuado en la zona sur - austral de Chile (Aguayo \& Gili 1984, Young et al. 1998).

Las primeras estimaciones para determinar la función de crecimiento en merluza de cola, de acuerdo con el modelo de von Bertalanffy, fueron efectuadas mediante métodos lineales y sin diferenciar sexos (Aguayo 1974, Aguayo \& Gili 1984), en tanto las más actualizadas han considerado longitudes individuales calculadas a edades pretéritas y ajustes no lineales (Young et al. 1998).

Los objetivos del presente trabajo fueron i) Estimar los parámetros de la función de crecimiento en longitud, para machos y hembras y establecer si existe crecimiento diferencial entre los sexos, ii) Estimar los parámetros de la función de crecimiento en peso para machos y hembras y, iii) Estimar la tasa instantánea de mortalidad natural.

\section{Material y métodos}

Los ejemplares de merluza de cola fueron obtenidos de las capturas efectuadas por barcos industriales de arrastre en la zona de la pesquería sur austral entre 1991 y 1996. Las medidas consideradas fueron la longitud total $(\mathrm{cm})$ y el peso total $(\mathrm{g})$ por sexo. Para el estudio de edad se recolectó el par de otolitos sagitta que fue guardado seco, individualmente, en sobres 
sellados con los respectivos datos y fecha de captura, hasta su posterior análisis.

\section{Crecimiento}

\section{Determinación de edad}

Se analizaron 6.786 ejemplares de merluza de cola $(M$. magellanicus) obtenidos durante los años 1991 a 1996. Del total de los ejemplares muestreados, 3.583 fueron hembras y 3.203 machos. Los otolitos sagitales se observaron con una lupa estereoscópica con aumento $10 \mathrm{X}$ provista de una reglilla graduada en 100 divisiones $(1 \mathrm{div}=0,01 \mathrm{~mm})$. Se midió tanto el radio del otolito como el de sus anillos de crecimiento desde el foco (núcleo) hasta el borde rostral de éstos.

Una zona de crecimiento en los otolitos se compone de una zona o banda opaca ancha y una zona o banda hialina delgada. En la estimación de edad se contaron y midieron los radios de los anillos hialinos anuales o annuli desde el foco hasta el borde rostral de cada annulus (Chugunova 1963, Chilton \& Beamish 1982). Para su observación, los otolitos fueron hidratados, al menos por 24 horas, antes de ser examinados con un estéreo microscopio. Luego, para resaltar los anillos de crecimiento, éstos fueron previamente teñidos con tinta negra permanente por su cara interna y sumergidos en una placa Petri con agua corriente e iluminados con luz reflejada o incidente. El análisis consistió en determinar el número de anillos hialinos o annuli (oscuros) y opacos (blanquecinos), registrar las medidas del radio del otolito (Ro) y el radio de cada anillo hialino ( $\mathrm{Rn}$ ), y determinar el tipo de borde. La validación de la periodicidad de la formación de los anillos anuales de crecimiento se realizó mediante el análisis mensual del borde, para lo cual se adoptó la nomenclatura de Dannevig (1933), consistente en asignar al borde del otolito la categoría de opaco ancho o angosto $(\mathrm{O}, \mathrm{o}) \mathrm{y}$ hialino ancho o angosto $(\mathrm{H}, \mathrm{h})$. La letra minúscula denota presencia incipiente del tipo de anillo en el borde.

La estimación de tallas promedios a cada edad se efectuó por medio de dos modalidades: i) edades pretéritas o retrocálculo y ii) edad actual.

El retrocálculo o determinación de tallas a edades pretéritas, estima la talla del pez a la cual se forma cada anillo hialino. En relación con ello, Francis (1990) recomienda el uso de regresiones ajustadas como la propuesta por Whitney \& Carlander (1956) fide Francis (1990), a fin de corregir desviaciones que se producen al calcular directamente de la ecuación de regresión lineal entre el radio del otolito y la longitud del pez. Esta corrección integra el radio del otolito ( $\mathrm{Rc})$, el anillo de crecimiento respectivo (Ri), la longitud del pez al momento de la captura (Lc) y las constantes de la regresión que se ajuste mejor a la relación entre el incremento en longitud del pez y el radio del otolito. La estimación de las tallas promedios a cada edad, mediante este método, involucra el retrocálculo para cada anillo de cada otolito.

La edad actual corresponde a la edad que presenta el pez al momento de la captura, la que es determinada a través del recuento de anillos hialinos o annuli en sus otolitos y, en este caso, cada individuo representa sólo una observación.

\section{Modelos de crecimiento}

El modelo de crecimiento utilizado, tanto en longitud como en peso, es el más usado en peces y corresponde a la ecuación de von Bertalanffy, cuya expresión integrada y linealizada ha sido propuesta por Beverton \& Holt (1957), según las siguientes ecuaciones que incluyen un factor de error aditivo $\left(\varepsilon_{t}\right)$ en razón a que el crecimiento se considera constante, esto es, como una función de la variable independiente:

Crecimiento en longitud

$$
\hat{L}_{t}=L_{\infty} *\left(1-e^{-K\left(t-t_{0}\right)}\right)+\varepsilon_{t}
$$

Crecimiento en peso

$$
\hat{W}_{t}=W_{\infty} *\left(1-e^{-K\left(t-t_{0}\right)}\right)^{b}+\varepsilon_{t}
$$

donde:

$\mathrm{L}_{\mathrm{t}}, \mathrm{W}_{\mathrm{t}}=$ longitud y peso promedio del pez a la edad $\mathrm{t}$

$\mathrm{L}_{\infty}, \mathrm{W}_{\infty}=$ longitud y peso promedio asintótico

$\mathrm{K}=$ constante metabólica (coeficiente de crecimiento)

$\mathrm{t}_{\mathrm{o}}=$ edad teórica a la longitud cero del pez

$\mathrm{t}=$ tiempo $($ edad $)$

$\mathrm{b}=$ coeficiente de la relación longitud-peso

Los parámetros de crecimiento $\mathrm{K}$ y $\mathrm{t}_{0}$ corresponden a los de crecimiento en longitud y el peso asintótico se estimó directamente de la relación longitud-peso correspondiente, de acuerdo a la siguiente expresión:

$$
W_{\infty}=a^{*}\left(L_{\infty}\right)^{b}
$$


El modelo de crecimiento se ajustó a los datos de edad y longitud individuales mediante ajuste no lineal. El procedimiento de minimización de cuadrados tiene la expresión:

$$
\mathrm{SSD}=\sum_{i=1}^{n}\left[\mathrm{Lt}-\mathrm{L}_{\infty}\left(1-\mathrm{e}^{-\mathrm{k}(\mathrm{t}-\mathrm{to})}\right)\right]^{2}
$$

donde:

SSD es la suma de cuadrados residuales

$\left(\sum_{t}\left(L_{t}-E\left(L_{t}\right)\right)^{2}\right)$.

El proceso de ajuste es iterativo y se consideraron como valores iniciales los parámetros de crecimiento estimados en forma específica para cada sexo por Ojeda et al. (1998).

La comparación de las funciones de crecimiento entre los sexos se realizó mediante la prueba $\mathrm{T}^{2}$ de Hotelling (Bernard 1981), pero con la formulación propuesta por Cerrato (1990), de acuerdo a la siguiente expresión:

$$
T^{2}=\frac{\left(P_{1}-P_{2}\right)^{\prime}}{S_{p 1-p 2}^{-1}\left(P_{1}-P_{2}\right)}
$$

donde:

$\left(P_{1}-P_{2}\right)^{\prime}=$ Vector transpuesto (vector fila) de $\left(\mathrm{P}_{1}-\right.$ $\mathrm{P}_{2}$ ), el cual está referido al vector diferencia de los parámetros de crecimiento (vector columna).

$S_{p_{1}-p_{2}}^{-1}=$ Inversa de la matriz de varianza-covarianza

del vector de diferencias de las estimaciones.

Considerando "Ho: Igualdad de vectores de los parámetros de crecimiento entre los sexos", la hipótesis Ho es aceptada si $\mathrm{T}^{2}$ calculado, es menor al valor crítico $\mathrm{T}_{\mathrm{o}}^{2}$, obtenido mediante la siguiente expresión:

$$
T_{0}^{2}=3 \frac{\left(N_{1}+N_{2}-6\right)}{\left(N_{1}+N_{2}-8\right)} F_{\alpha}\left(3, N_{1}+N_{2}-6\right)
$$

En la ecuación $\mathrm{F}$ corresponde al percentil de la distribución de Fisher con 3 y $\mathrm{N}_{1}+\mathrm{N}_{2}-6$ grados de libertad. La prueba considera como hipótesis nula que los vectores de los parámetros de crecimiento son iguales y la rechaza cuando el $T_{0}^{2}$ calculado es mayor que el valor crítico.

Un segundo método de comparación fue el análisis de la suma de cuadrados residuales sugerida por Chen et al. (1992), cuya expresión es la siguiente:

$$
F=\frac{\frac{R S S_{p}-R S S_{s}}{3(K-1)}}{\frac{R S S_{s}}{N-3 K}}
$$

donde:

$\mathrm{N}=$ Número total de muestras

$\mathrm{K}=$ Número de grupos en comparación

RSSs = Suma de cuadrados residuales

RSSp = Suma de cuadrados residuales del ajuste agrupado

\section{Tasa instantánea de mortalidad natural}

En la estimación de la tasa instantánea de mortalidad natural se utilizaron métodos bioanalógicos que consideran valores de parámetros de crecimiento y ambientales y métodos de curvas de capturas sobre la base de la composición por edades y longitudes.

El parámetro $\mathrm{M}$ es complejo de estimar, dado que generalmente la información que se dispone corresponde a períodos donde ya existe mortalidad por pesca $(\mathrm{F})$, es decir, hay una pesquería en desarrollo y, por lo tanto, lo que se calcula con el segundo grupo de métodos es la mortalidad total Z. Sin embargo, cuando la información de composición por edades o longitudes del pez es de un período anterior a la pesquería, o a lo más cuando ésta es incipiente ( $\mathrm{F}$ es pequeño), se logran estimar valores más precisos de $\mathrm{M}$.

En el caso de los métodos bioanalógicos, las fórmulas analíticas provienen de relaciones teóricas entre los diferentes parámetros o bien, se deriva de regresiones entre $\mathrm{M}$ y uno o más parámetros de crecimiento. Estos modelos tienen como principal ventaja el que requieren una mínima cantidad de datos (Sparre \& Venema 1998).

\section{Métodos bioanalógicos}

El supuesto básico de este grupo de métodos es la relación entre la mortalidad natural y uno o más parámetros biológicos, ambientales u otros. 


\section{Método de Taylor (1960)}

La expresión propuesta por Taylor para estimar M es la siguiente:

$$
M=\frac{2,996}{A_{0,95}-T r}
$$

donde $\mathrm{A}_{0,95}$ corresponde a la edad límite, que es aquella cuando un individuo alcanza una longitud correspondiente al $95 \%$ de $\mathrm{L}_{\infty}$, y $\operatorname{Tr}$ es la edad de reclutamiento a la población. La edad límite se deriva de la ecuación de crecimiento de von Bertalanffy, siendo:

$$
A_{0,95}=\frac{2,996}{K}+t_{0}
$$

donde $\mathrm{K}$ es el coeficiente de catabolismo y $\mathrm{t}_{\mathrm{o}}$ es la edad teórica a la cual el pez tiene una longitud cero.

\section{Método de Alagaraja (1984)}

Este método define a la longevidad como la edad a la que el $99 \%$ de una cohorte habría muerto si hubiera estado expuesta solamente a mortalidad natural, esto es, que $\mathrm{Z}=\mathrm{M}$. De esta manera, la mortalidad natural para el $1 \%$ de los sobrevivientes es estimada por la expresión:

$$
M=\frac{\ln (0,01)}{T_{\max }-T r}
$$

$\mathrm{T}_{\max }$ corresponde a la edad máxima o límite que se calculará como $\mathrm{A}_{0,95} \mathrm{y} \operatorname{Tr}$ a la edad de reclutamiento.

\section{Método de Pauly (1980)}

Este método proporciona un estimado de $\mathrm{M}$, sobre la base de la relación que existe entre este parámetro y los parámetros de crecimiento, en especial, el parámetro K de la ecuación de crecimiento de von Bertalanffy. Además, considera la temperatura $\left(\mathrm{T}^{\circ}\right)$ del hábitat de la especie, por el efecto que tiene sobre el coeficiente $\mathrm{K}$ y la longitud asintótica $\left(\mathrm{L}_{\infty}\right)$. Las relaciones fueron establecidas por el autor, considerando los datos de 175 poblaciones diferentes.

La ecuación que representa esta relación es la siguiente:

$\ln \mathrm{M}=-0,0152-0,279 * \ln \mathrm{L}_{\infty}+0,6543 * \ln \mathrm{K}+0,4634$ $* \ln \mathrm{T}$

Para el caso de peces que forman cardúmenes, como lo merluza de cola, Pauly sugiere que el valor estimado de M debe considerarse un 20\% menor. En esta situación la ecuación queda de la forma siguiente:

$\mathrm{M}=0,8 * \exp \left[0,0152-0,279 * \ln \mathrm{L}_{\infty}+0,6543 * \ln \mathrm{K}+\right.$ $0,4634 * \ln \mathrm{T}]$

\section{Método de Alverson \& Cartney (1975)}

Este método estima $\mathrm{M}$ a partir de la siguiente expresión:

$$
M=\frac{b K}{\left[\exp \left(K\left(t_{c r i t}-t_{0}\right)-1\right)\right]}
$$

donde $\mathrm{b}$ es el exponente de la relación longitud-peso y $\mathrm{K}$ y $\mathrm{t}_{0}$ son los parámetros de la ecuación de crecimiento de von Bertalanffy. Por otra parte, la edad crítica ( $\left.t_{\text {crit }}\right)$ de acuerdo con Csirke (1980) se calcula mediante la expresión siguiente:

$$
\text { tcrit }=\mathrm{t}_{0}+\frac{1}{K} * \ln \left[\frac{b K}{M}+1\right]
$$

\section{Variabilidad de los estimados de $\mathrm{M}$}

La variabilidad de los valores de $\mathrm{M}$, estimados mediante los métodos bioanalógicos, se realizó mediante remuestreo bootstrap (Efron 1985) evaluado numéricamente por medio de ensayos de Monte Carlo que básicamente consistió en generar muestras aleatorias desde una distribución de probabilidades conocidas. La variabilidad se midió en términos de la desviación estándar del análisis de Monte Carlo y para asegurar la estabilidad de los estimados, se utilizaron 1000 muestras. La desviación estándar se estimó como:

$$
\text { d.e. }(\mathrm{M})=\sqrt{\frac{\sum_{i=1}^{m}(M i-\bar{M})^{2}}{m-1}}
$$

donde $\mathrm{m}$ es el número de muestras (1000) y Mi es la mortalidad natural de la muestra i y la mortalidad natural promedio es:

$$
\bar{M}=\frac{\sum_{i=1}^{m} M i}{m}
$$

En la obtención de las muestras aleatorias de M, se emplearon los errores estándares de los parámetros de las ecuaciones de los diferentes métodos citados. 


\section{Métodos de la curva de captura linealizada}

La estimación más precisa de $\mathrm{M}$ es la obtenida en ausencia de mortalidad por pesca o cuando ésta es ínfima, de manera que el valor de $\mathrm{Z}$ responde casi exclusivamente a $\mathrm{M}$.

\section{Curva de captura linealizada basada en composición por edades}

Chapman \& Robson (1960) y Robson \& Chapman (1961) proponen la siguiente ecuación de la captura linealizada con intervalos de tiempo constante, donde la pendiente es $\mathrm{Z}$.

$$
\ln [\mathrm{C}(\mathrm{t}, \mathrm{t}+\Delta \mathrm{t})]=\mathrm{g}-\mathrm{Z} * \mathrm{t}
$$

donde $\mathrm{C}(\mathrm{t}, \mathrm{t}+\Delta \mathrm{t})=$ captura entre dos edades de una cohorte; $\mathrm{g}=$ intercepto; $\mathrm{t}=$ edad; $\mathrm{Z}=$ pendiente de la línea de regresión que en ausencia de mortalidad por pesca equivale a $\mathrm{M}$.

En la aplicación de este método se usó información de composición por edades de capturas en los primeros años de la pesquería, donde esta especie aparecía como incidental en la captura de arrastre de fondo de Merluccius australis (merluza del sur o austral) y Genypterus blacodes (congrio dorado) con volúmenes bajos de capturas (Aguayo et al. 1990, 1991).

\section{Regresión lineal de curva de captura basada en composición por tallas}

En el caso de la transformación lineal de la curva de captura basada en estructura de talla, la información de composición por talla de las capturas utilizadas correspondió a las capturas efectuadas en un crucero de investigación realizado por el Instituto de Fomento Pesquero en 1972 (Pantoja et al. 1973), cinco años antes que se iniciara la pesquería demersal en la zona, esto es, con una mortalidad por pesca incipiente.

En la estimación de $M$ se aplicó la ecuación de Jones \& van Zalinge (1981) cuya formulación es la siguiente:

$$
\ln \mathrm{C}\left(\mathrm{L}, \mathrm{L}_{\infty}\right)=a+\frac{Z}{K} * \ln \left(L_{\infty}-L\right)
$$

donde:

C $\left(\mathrm{L}, \mathrm{L}_{\infty}\right)$, representa la captura acumulada de peces de talla L y mayores. La pendiente de la línea de regresión es $\mathrm{Z} / \mathrm{K}$, de manera que $\mathrm{Z}=\mathrm{K} *$ pendiente.
Para los métodos de curva de captura basados en edad y talla, la variabilidad de $\mathrm{M}$ estará dada por el error estándar del ajuste de las respectivas regresiones lineales de cada método. Luego de obtener los estimados de $\mathrm{M}$ y sus errores estándares mediante los diferentes métodos, se calculó un valor de M promedio, ponderado por el inverso de la varianza de cada estimado:

$$
\widehat{M}=\frac{\sum_{j=1}^{n} w j M j}{\sum_{j=1}^{n} w j}
$$

donde $\mathrm{M}_{\mathrm{j}}$ corresponde a la estimación de mortalidad natural por el método $\mathrm{j}(=1,2 \ldots, \mathrm{n}) \mathrm{y} \mathrm{w}_{\mathrm{j}}$ es el inverso de la varianza $\left(w_{j}=1 / s_{j}^{2}\right)$.

\section{Resultados}

El número de annuli observados en los otolitos sagitta fue de 16 , sin embargo, debido a la baja presencia de otolitos en tallas mayores, sólo se consideró hasta el annulus 14 en ambos sexos. En la Fig. 1 se presenta una fotografía de un otolito sagitta iluminado con luz incidente o reflejada correspondiente a un ejemplar de $89 \mathrm{~cm}$ de longitud total con 13 años, donde se puede observar en forma nítida los annuli o anillos hialinos (H) de crecimiento lento, alternados con anillos opacos

(O) de crecimiento rápido.

\section{Crecimiento}

\section{Determinación de edad}

Periodicidad en la formación de los anillos de crecimiento

La distribución mensual de los porcentajes de bordes en los otolitos muestra que las mayores frecuencias de bordes hialinos se presentan desde junio a agosto con un máximo en julio, mientras que el borde opaco predomina durante los meses de septiembre a febrero con máximo en diciembre-enero (Fig. 2). Esto señala la formación de un anillo hialino y uno opaco en el transcurso de un año. 


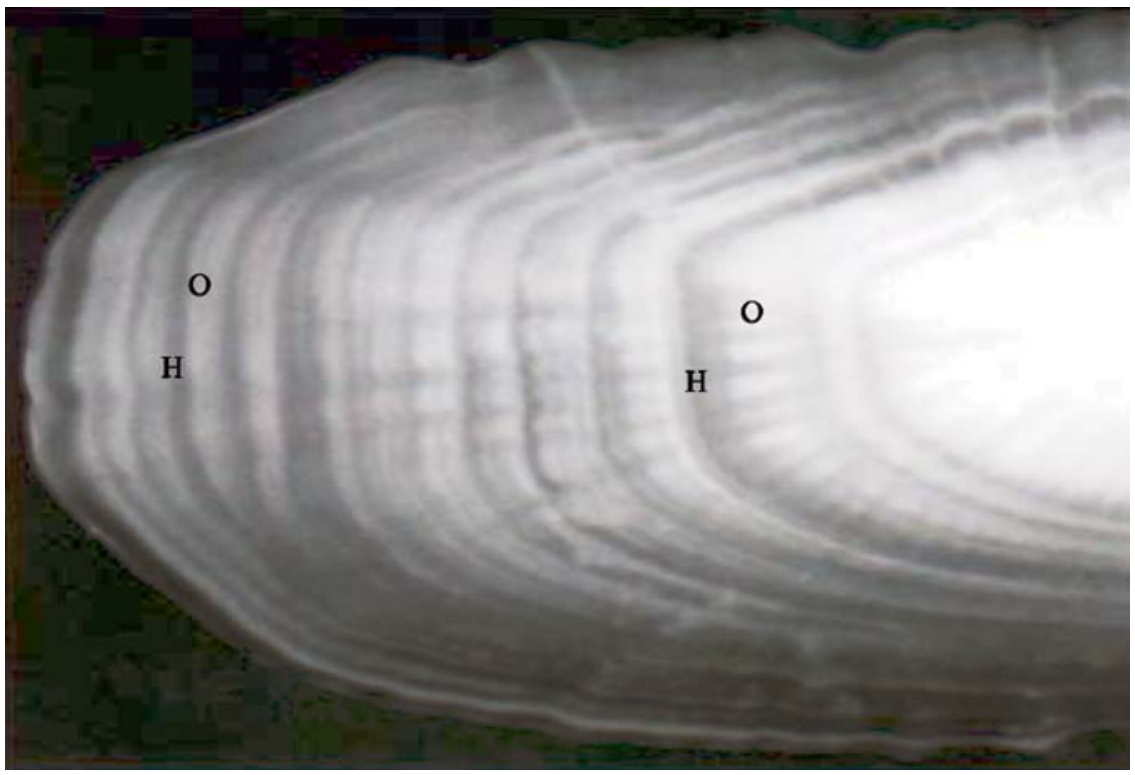

\section{Figura 1}

Fotografía de un otolito sagitta de $M$. magellanicus, correspondiente a un ejemplar de 13 años ( $\mathrm{H}=$ anillos hialinos, zonas oscuras; $\mathrm{O}=$ anillos opacos, zonas blancas)

Fotography of a sagitta otolith of a 13-year-old M. magellanicus

( $\mathrm{H}=$ hyaline rings, dark zones; $\mathrm{O}=$ opaque rings, white zones $)$

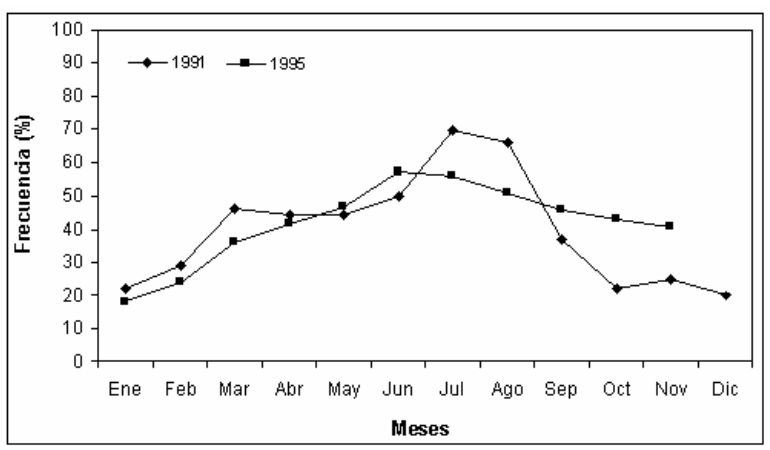

Figura 2

Frecuencia mensual de bordes hialinos en los otolitos sagitta de M. magellanicus durante los años 1991 y 1995

Monthly frequency of the hyalines edges in the sagitta otolith of M. magellanicus during the 1991 and 1995 years
Proporcionalidad entre la longitud del pez $\left(L_{p}\right)$ y el tamaño del otolito $\left(\mathbf{R}_{\mathbf{0}}\right)$

Las relaciones potenciales ajustadas en forma no lineal, presentaron el mejor ajuste y fueron las siguientes: (Tabla 1, Figs. 3 y 4):

$L_{p}=0,27429 \cdot R_{o}^{1,23573}$ (machos) $(\mathrm{R}=0,997)$

$L_{p}=0,25367 \cdot R_{o}^{1,25525}$ (hembras) $(\mathrm{R}=0,996)$

$L_{p}=0,2559 \cdot R_{o}^{1,2524} \quad($ combinados $)(\mathrm{R}=0,918)$

\section{Crecimiento en longitud}

Estimación de crecimiento mediante retrocálculo o longitudes a edades pretéritas

En los machos, el número de pares ordenados correspondientes a los anillos individuales utilizados para el retrocálculo fue de 17.660 , mientras que para las hembras se elevó a 20.726, observándose una baja presencia de ejemplares en edades superiores a los 12 años, en sexos combinados. 
Tabla 1

Valores de la regresión potencial entre la longitud total del pez y el radio del otolito en M. magellanicus

Value of the potential regression between the total length of the fish and the otolith ratio in M. magellanicus

\begin{tabular}{lcccccc}
\hline & \multicolumn{2}{c}{ MACHOS } & \multicolumn{2}{c}{ HEMBRAS } & \multicolumn{2}{c}{ AMBOS } \\
& Intercepto & Pendiente & Intercepto & Pendiente & Intercepto & Pendiente \\
\hline Coeficientes & 0,27429 & 1,23573 & 0,25367 & 1,25525 & 0,25590 & 1,25240 \\
Error estándar & 0,01309 & 0,01060 & 0,01195 & 0,01035 & 0,00857 & 0,00739 \\
L. Inf. 95\% & 0,24863 & 1,21496 & 0,23024 & 1,23495 & 0,23910 & 1,23791 \\
L. Sup. 95\% & 0,29994 & 1,26651 & 0,27710 & 1,27555 & 0,27269 & 1,26688 \\
Observaciones & \multicolumn{2}{c}{2841} & \multicolumn{2}{c}{3335} & & \multicolumn{2}{c}{6176} \\
\hline
\end{tabular}

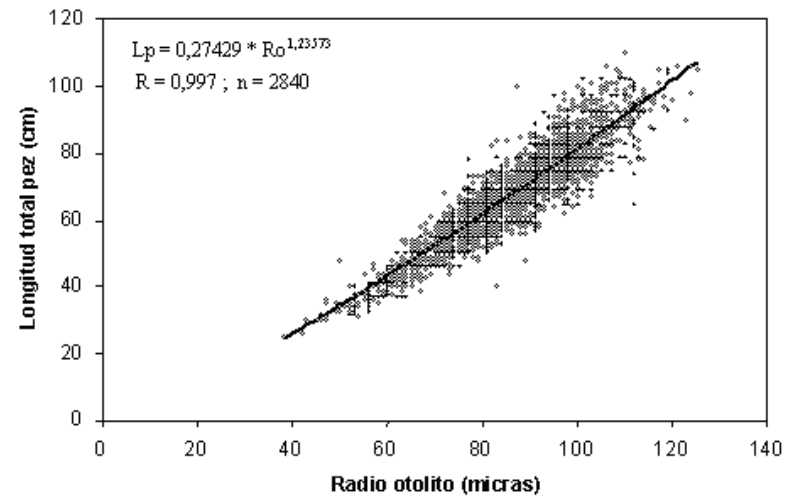

Figura 3

Relación entre la longitud total del pez y el radio del otolito, determinada para machos de M. magellanicus

Relation between total length of the fish and the otolith radius determined for males of M. magellanicus

Las longitudes promedios retrocalculadas y las tasas de crecimiento correspondientes se presentan en la Tabla 2 para los machos y en la Tabla 3 para las hembras, donde se observa que los valores calculados son muy similares hasta la edad 4 y que a partir de la edad 5 se presentan las diferencias de crecimiento entre los sexos, alcanzando las hembras tallas mayores a las respectivas edades.

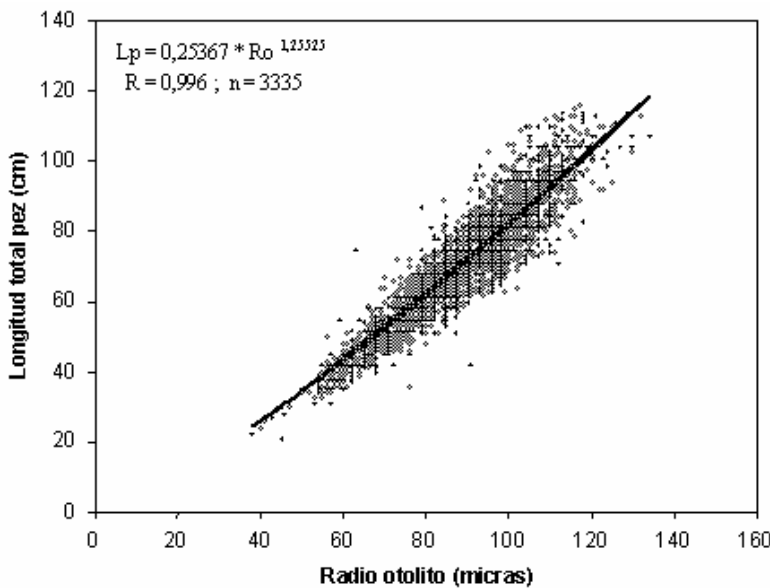

Figura 4

Relación entre la longitud total del pez y el radio del otolito, determinada para hembras de M. magellanicus

Relation between total length of the fish and the otolith radius determined for females of M. magellanicus

El método de ajuste no lineal efectuado sobre la base de los datos individuales de longitud retrocalculada basada en los anillos de crecimiento, entregó las expresiones siguientes para hembras, machos y sexos combinados (Fig. 5):

Hembras: $\mathrm{L}_{\mathrm{t}}=105,5\left[1-\mathrm{e}^{-0,16704(\mathrm{t}+0,81273)}\right] \quad(\mathrm{R}=0,948)$

Machos: $\mathrm{L}_{\mathrm{t}}=95,6\left[1-\mathrm{e}^{-0,19127(\mathrm{t}+0,78882)}\right] \quad(\mathrm{R}=0,945)$

Combinado: $\mathrm{L}_{\mathrm{t}}=101,3\left[1-\mathrm{e}^{-0,17588(\mathrm{t}+0,81239)}\right](\mathrm{R}=0,946)$ 
Tabla 2

\section{Longitudes promedios retrocalculadas y estimadas para cada anillo y valores de estadísticos asociados para machos de M. magellanicus}

Backcalculated mean lengths and estimated for each annuli and statistical values for males of $M$. magellanicus

\begin{tabular}{|c|c|c|c|c|c|c|c|c|}
\hline \multirow{2}{*}{$\begin{array}{l}\text { Edad } \\
\text { (años) }\end{array}$} & \multirow{2}{*}{$\begin{array}{l}\text { Long. prom. } \\
\text { retroc. }(\mathrm{cm})\end{array}$} & \multirow{2}{*}{$\begin{array}{c}\text { Error } \\
\text { estándar }\end{array}$} & \multirow{2}{*}{$\begin{array}{c}\mathrm{N}^{\circ} \\
\text { anillos } \\
\text { medidos }\end{array}$} & \multicolumn{2}{|c|}{ Intervalos $(95 \%)$} & \multirow{2}{*}{$\begin{array}{l}\text { Long. estim. } \\
\text { corregidas } \\
\text { (cm) }\end{array}$} & \multicolumn{2}{|c|}{ Tasas } \\
\hline & & & & $\begin{array}{l}\text { Lim. inf. } \\
(\mathrm{cm})\end{array}$ & $\begin{array}{l}\text { Lim. sup. } \\
\text { (cm) }\end{array}$ & & Incr. absol. & Incr.relat. \\
\hline 1 & 24 & 4,0 & 3100 & 12 & 37 & 25,2 & & \\
\hline 2 & 39 & 4,3 & 3115 & 27 & 59 & 38,1 & 12,86 & 50,99 \\
\hline 3 & 49 & 5,1 & 2850 & 34 & 70 & 48,4 & 10,31 & 27,08 \\
\hline 4 & 57 & 5,9 & 2481 & 39 & 77 & 56,7 & 8,27 & 17,09 \\
\hline 5 & 64 & 6,5 & 1971 & 45 & 85 & 63,3 & 6,63 & 11,7 \\
\hline 6 & 69 & 6,7 & 1533 & 49 & 90 & 68,6 & 5,32 & 8,4 \\
\hline 7 & 74 & 7,2 & 1041 & 52 & 94 & 72,9 & 4,26 & 6,22 \\
\hline 8 & 78 & 8,1 & 632 & 56 & 99 & 76,3 & 3,42 & 4,69 \\
\hline 9 & 82 & 8,7 & 313 & 60 & 105 & 79 & 2,74 & 3,59 \\
\hline 10 & 87 & 8,4 & 131 & 65 & 105 & 81,2 & 2,2 & 2,78 \\
\hline 11 & 90 & 7,8 & 55 & 72 & 108 & 83 & 1,76 & 2,17 \\
\hline 12 & 92 & 7,7 & 20 & 74 & 104 & 84,4 & 1,41 & 1,7 \\
\hline 13 & 95 & 7,5 & 5 & 82 & 102 & 85,5 & 1,13 & 1,34 \\
\hline 14 & 96 & 9,9 & 3 & 84 & 102 & 86,4 & 0,91 & 1,06 \\
\hline
\end{tabular}

Tabla 3

Longitudes promedios retrocalculadas y estimadas para cada anillo y valores de estadísticos asociados para hembras de $M$. magellanicus

Backcalculated mean lengths and estimated for each annuli and statistical values for females of M. magellanicus

\begin{tabular}{|c|c|c|c|c|c|c|c|c|}
\hline \multirow{2}{*}{$\begin{array}{l}\text { Edad } \\
\text { (años) }\end{array}$} & \multirow{2}{*}{$\begin{array}{l}\text { Long. prom. } \\
\text { retroc. }(\mathrm{cm})\end{array}$} & \multirow{2}{*}{$\begin{array}{c}\text { Error } \\
\text { estándar }\end{array}$} & \multirow{2}{*}{$\begin{array}{c}\mathrm{N}^{\circ} \\
\text { anillos } \\
\text { medidos }\end{array}$} & \multicolumn{2}{|c|}{ Intervalos $(95 \%)$} & \multirow{2}{*}{$\begin{array}{l}\text { Long. estim. } \\
\text { corregidas } \\
(\mathrm{cm})\end{array}$} & \multicolumn{2}{|c|}{ Tasas } \\
\hline & & & & $\begin{array}{l}\text { Lim. inf. } \\
(\mathrm{cm})\end{array}$ & $\begin{array}{l}\text { Lim. sup. } \\
\text { (cm). }\end{array}$ & & Incr. absol. & Incr.relat. \\
\hline 1 & 23 & 4,3 & 3446 & 14,6 & 31,4 & 23,8 & & \\
\hline 2 & 39 & 4,4 & 3503 & 30,4 & 47,6 & 37,5 & 13,75 & 57,91 \\
\hline 3 & 49 & 5,3 & 3182 & 38,6 & 59,4 & 48,8 & 11,31 & 30,15 \\
\hline 4 & 58 & 6,1 & 2857 & 46,0 & 70,0 & 58,1 & 9,3 & 19,04 \\
\hline 5 & 65 & 6,8 & 2399 & 51,7 & 78,3 & 65,7 & 7,64 & 13,15 \\
\hline 6 & 71 & 7,2 & 1960 & 56,9 & 85,1 & 72,0 & 6,28 & 9,56 \\
\hline 7 & 76 & 7,8 & 1495 & 60,7 & 91,3 & 77,2 & 5,17 & 7,17 \\
\hline 8 & 81 & 8,5 & 961 & 64,3 & 97,7 & 81,4 & 4,25 & 5,5 \\
\hline 9 & 86 & 8,9 & 533 & 68,6 & 103,4 & 84,9 & 3,49 & 4,29 \\
\hline 10 & 91 & 9,6 & 254 & 72,2 & 109,8 & 87,8 & 2,87 & 3,38 \\
\hline 11 & 94 & 9,8 & 103 & 74,8 & 113,2 & 90,2 & 2,36 & 2,69 \\
\hline 12 & 97 & 9,8 & 45 & 77,8 & 116,2 & 92,1 & 1,94 & 2,15 \\
\hline 13 & 98 & 6,8 & 16 & 84,7 & 111,3 & 93,7 & 1,59 & 1,73 \\
\hline 14 & 101 & 8,3 & 7 & 84,7 & 117,3 & 95,0 & 1,31 & 1,4 \\
\hline
\end{tabular}




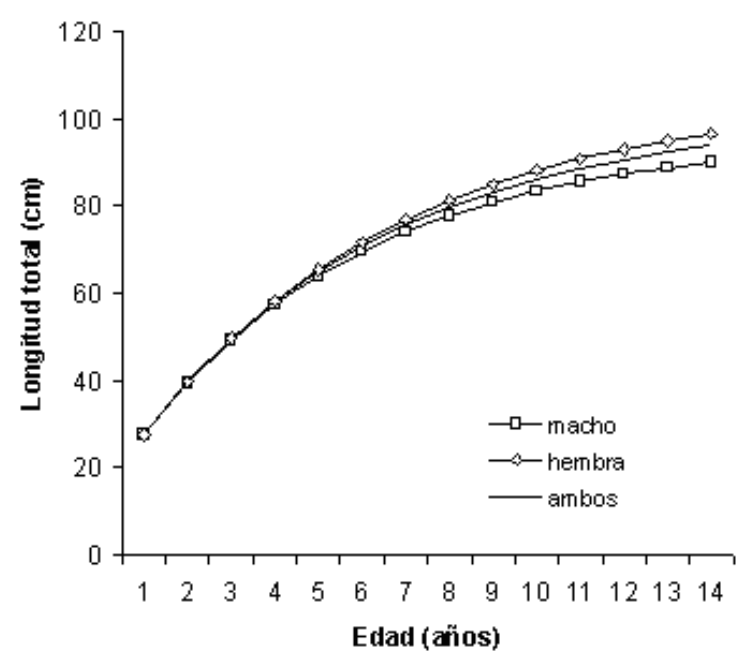

Figura 5

Curvas de crecimiento en longitud obtenida por ajuste no lineal, para longitud-edad retrocalculada, en machos, hembras y ambos sexos de $M$. magellanicus

Growth curves in length by non linear fit for back calculated age length in males, females, and both sexes of $M$. magellanicus

La longitud asintótica es mayor en las hembras. En la Tabla 6 se presentan los valores de estos parámetros con sus respectivos errores y límites de confianza (95\%). Por otra parte, en las Tablas 7, 8 y 9 se entregan las longitudes promedios a la edad calculadas con las respectivas ecuaciones de crecimiento.

\section{Comparación de parámetros de crecimiento entre sexos}

El análisis multivariado o prueba T2 de Hotelling (Cerrato 1990) señala que la diferencia entre los vectores columna de los parámetros de crecimiento es:

$$
\delta=\left|\begin{array}{l}
-9,82 \\
0,0242 \\
0,0239
\end{array}\right|
$$

y la matriz de covarianza agrupada y su inversa corresponde a los valores de la Tabla 4.

\section{Tabla 4}

Matriz de covarianza agrupada y su inversa en el crecimiento de machos y hembras de $M$. magellanicus

Matrix of covariance grouped and its inverse in the growth of males and females of M. magellanicus

\begin{tabular}{cccc}
\hline & 0,5319917 & $-0,0019109$ & $-0,012143$ \\
& $-0,001911$ & $7,225 \mathrm{E}-06$ & $5,016 \mathrm{E}-05$ \\
$-0,012143$ & $5,024 \mathrm{E}-05$ & 0,0004345 \\
\hline & & & \\
$\mathrm{U}-1=$ & 306,87511 & 30522,226 & $-1123,321$ \\
& $-1144,617$ & $-487677,5$ & 26629,773 \\
\hline
\end{tabular}

Finalmente, el estadístico $\mathrm{T}^{2}$ observado tiene un valor de 518,8 mayor que el valor crítico $T_{o}^{2}=25,59$ para un nivel $\alpha=0.05$, lo que significa que no se acepta la hipótesis nula $\delta=0$; esto es, el conjunto de parámetros de crecimiento de machos y hembras son significativamente diferentes.

Por otra parte, el resultado del análisis de la suma de cuadrados residuales (RSS) también indica que las muestras comparadas originan estimados de crecimiento estadísticamente diferentes, como se presenta en la Tabla 5 .

Por lo tanto, la comparación de las curvas de crecimiento mediante los dos métodos aplicados entrega como resultado la existencia de crecimiento diferencial entre los sexos de M. magellanicus, siendo mayor en las hembras.

\section{Tabla 5}

Comparación de suma de cuadrados residuales de machos y hembras de $M$. magellanicus

Comparison of residual squares sum of males and females of M. magellanicus

\begin{tabular}{lc}
\hline Categorías & RSS \\
\hline RSS de machos & 553894,55 \\
RSS de hembras & 733167,33 \\
RSSs suma de residuales de sexos & 1287061,88 \\
combinados & 1309347,46 \\
RSSp residuales de la muestra total & 221,51 \\
F calculado & 2,6 \\
$\mathrm{~F}_{((3 \mathrm{~K}-1) ; \mathrm{N}-3 \mathrm{~K}),(\alpha=5 \%)}$ & \\
\hline
\end{tabular}


Estimación de crecimiento mediante longitudes a la edad actual

El ajuste no lineal realizado con los datos de longitud a la edad actual, entregó las siguientes funciones de crecimiento para hembras, machos y sexos combinados (Fig. 6).

Hembras: $\mathrm{Lt}=127,97 \mathrm{~cm}\left[1-\mathrm{e}^{-0,09733(\mathrm{t}+2,43477)}\right](\mathrm{R}=0,843)$

Machos: $\mathrm{Lt}=111,87 \mathrm{~cm}\left[1-\mathrm{e}^{-0,11687(\mathrm{t}+2,32667)}\right](\mathrm{R}=0,856)$

Combinados: $\mathrm{Lt}=124,4 \mathrm{~cm}\left[1-\mathrm{e}^{-0.09872(\mathrm{t}+2,50710}\right](\mathrm{R}=0,850)$
En las Tablas 10 y 11, se entregan las longitudes promedios estimadas a la edad actual con sus límites de confianza (95\%), así como las tasas de incremento, para machos, hembras y sexos combinados de merluza de cola, respectivamente.

Los valores de estos parámetros de crecimiento, asi como los estadísticos asociados, con sus respectivos límites de confianza por sexo, se presentan en la Tabla 12.

Tabla 6

Valores de los parámetros de crecimiento en longitud para M. magellanicus, estimados mediante ajuste no lineal de la función de von Bertalanffy (longitud retrocalculada)

Values of the growth length parameters for M. magellanicus, estimated by nonlinear regression fit of the von Bertalanffy function (back calculated length)

\begin{tabular}{cccccc}
\hline Sexo & Parámetros & Valor & Error & \multicolumn{2}{c}{ Intervalos (95\%) } \\
\cline { 5 - 6 } & & estimado & estándar & L. Inferior & L. Superior \\
\hline \multirow{3}{*}{ Machos } & $\mathrm{L}_{\infty}$ & 95,65 & 0,51292 & 94,64 & 96,66 \\
& $\mathrm{~K}$ & 0,19127 & 0,00248 & 0,18641 & 0,19613 \\
& $\mathrm{t}_{\mathrm{O}}$ & $-0,78882$ & 0,01707 & $-0,82228$ & $-0,75536$ \\
\hline \multirow{3}{*}{ Hembrannyyy} & $\mathrm{L}_{\infty}$ & 105,5 & 0,60483 & 104,31 & 106,69 \\
& $\mathrm{~K}$ & 0,16704 & 0,00214 & 0,16285 & 0,17123 \\
& $\mathrm{t}_{\mathrm{O}}$ & $-0,81273$ & 0,01680 & $-0,84566$ & $-0,77980$ \\
\hline \multirow{3}{*}{ Ambos } & $\mathrm{L}_{\infty}$ & 101,3 & 0,40764 & 100,5 & 102,1 \\
& $\mathrm{~K}$ & 0,17588 & 0,00163 & 0,17269 & 0,17907 \\
& $\mathrm{t}_{\mathrm{O}}$ & $-0,81239$ & 0,01234 & $-0,83658$ & $-0,7882$ \\
\hline
\end{tabular}

Tabla 7

Longitud promedio a la edad actual calculada mediante la ecuación de von Bertalanffy en machos de M. magellanicus

Mean length at age calculated by the von Bertalanffy equation in males of M. magellanicus

\begin{tabular}{|c|c|c|c|c|c|}
\hline \multirow{2}{*}{$\begin{array}{l}\text { Edad } \\
\text { (años) }\end{array}$} & \multirow{2}{*}{$\begin{array}{l}\text { Longitud } \\
\text { promedio } \\
\text { calculada } \\
(\mathrm{cm})\end{array}$} & \multicolumn{2}{|c|}{ Intervalos de confianza (95\%) } & \multicolumn{2}{|c|}{ Tasas } \\
\hline & & $\begin{array}{l}\text { Long. inferior } \\
(\mathrm{cm})\end{array}$ & $\begin{array}{l}\text { Long. superior } \\
(\mathrm{cm})\end{array}$ & $\begin{array}{c}\text { Incr. absoluto } \\
(\mathrm{cm})\end{array}$ & $\begin{array}{c}\text { Incr. relativo } \\
(\%)\end{array}$ \\
\hline 1 & 27,7 & 27,3 & 28,2 & 27,7 & \\
\hline 2 & 39,5 & 38,7 & 40,4 & 11,8 & 42,60 \\
\hline 3 & 49,3 & 48,2 & 50,4 & 9,8 & 24,81 \\
\hline 4 & 57,4 & 56,1 & 58,6 & 8,1 & 16,43 \\
\hline 5 & 64,0 & 62,7 & 65,4 & 6,6 & 11,50 \\
\hline 6 & 69,5 & 68,1 & 71,0 & 5,5 & 8,59 \\
\hline 7 & 74,1 & 72,6 & 75,5 & 4,6 & 6,62 \\
\hline 8 & 77,8 & 76,4 & 79,3 & 3,7 & 4,99 \\
\hline 9 & 80,9 & 79,5 & 82,4 & 3,1 & 3,98 \\
\hline 10 & 83,5 & 82,1 & 84,9 & 2,6 & 3,21 \\
\hline 11 & 85,6 & 84,2 & 87,0 & 2,1 & 2,51 \\
\hline 12 & 87,4 & 86,0 & 88,7 & 1,8 & 2,10 \\
\hline 13 & 88,8 & 87,4 & 90,1 & 1,4 & 1,60 \\
\hline 14 & 90,0 & 88,7 & 91,3 & 1,2 & 1,35 \\
\hline
\end{tabular}


Tabla 8

Longitud promedio a la edad actual calculada mediante la ecuación de von Bertalanffy en hembras de M. magellanicus

Mean length at present age in females calculated by the von Bertalanffy equation in M. magellanicus

\begin{tabular}{rccccc}
\hline \multirow{2}{*}{$\begin{array}{r}\text { Edad } \\
\text { (años) }\end{array}$} & $\begin{array}{c}\text { Longitud } \\
\text { promedio }\end{array}$ & \multicolumn{2}{c}{ Intervalos (95\%) } & \multicolumn{2}{c}{ Tasas } \\
\cline { 3 - 6 } & calculada & Long. inferior & Long. superior & Incr. absoluto & Incr. relativo \\
& $(\mathrm{cm})$ & $(\mathrm{cm})$ & $(\mathrm{cm})$ & $(\mathrm{cm})$ & $(\%)$ \\
\hline 1 & 27,6 & 27,1 & 28,0 & 27,6 & 43,5 \\
2 & 39,6 & 38,7 & 40,4 & 12,0 & 25,5 \\
3 & 49,7 & 48,5 & 50,8 & 10,1 & 17,3 \\
4 & 58,3 & 56,9 & 59,6 & 8,6 & 12,4 \\
5 & 65,5 & 64,0 & 67,0 & 7,2 & 9,5 \\
6 & 71,7 & 70,1 & 73,3 & 6,2 & 7,3 \\
7 & 76,9 & 75,2 & 78,5 & 5,2 & 5,7 \\
8 & 81,3 & 79,6 & 83,0 & 4,4 & 4,6 \\
9 & 85,0 & 83,3 & 86,7 & 3,7 & 3,8 \\
10 & 88,2 & 86,5 & 89,8 & 3,2 & 3,0 \\
11 & 90,8 & 89,2 & 92,5 & 2,6 & 2,5 \\
12 & 93,1 & 91,4 & 94,7 & 2,3 & 2,0 \\
13 & 95,0 & 93,4 & 96,6 & 1,9 & 1,7 \\
14 & 96,6 & 95,0 & 98,2 & 1,6 & \\
\hline
\end{tabular}

Tabla 9

Longitud promedio a la edad actual calculada mediante la ecuación de von Bertalanffy para ambos sexos en M. magellanicus Mean length at present age calculated by the von Bertalanffy equation for both sexes in M. magellanicus.

\begin{tabular}{|c|c|c|c|c|c|}
\hline \multirow{2}{*}{$\begin{array}{l}\text { Edad } \\
\text { (años) }\end{array}$} & \multirow{2}{*}{$\begin{array}{c}\text { Longitud } \\
\text { promedio } \\
\text { calculada } \\
(\mathrm{cm})\end{array}$} & \multicolumn{2}{|c|}{ Intervalos de confianza $(95 \%)$} & \multicolumn{2}{|c|}{ Tasas } \\
\hline & & $\begin{array}{l}\text { Long. inferior } \\
(\mathrm{cm})\end{array}$ & $\begin{array}{l}\text { Long. superior } \\
(\mathrm{cm})\end{array}$ & $\begin{array}{c}\text { Incr. absoluto } \\
(\mathrm{cm})\end{array}$ & $\begin{array}{c}\text { Incr. relativo } \\
(\%) \\
\end{array}$ \\
\hline 1 & 27,7 & 27,3 & 28,0 & 27,7 & \\
\hline 2 & 39,5 & 38,9 & 40,1 & 11,9 & 42,96 \\
\hline 3 & 49,5 & 48,7 & 50,3 & 10,0 & 25,20 \\
\hline 4 & 57,8 & 56,9 & 58,8 & 8,4 & 16,88 \\
\hline 5 & 64,9 & 63,8 & 65,9 & 7,0 & 12,12 \\
\hline 6 & 70,7 & 69,6 & 71,8 & 5,9 & 9,06 \\
\hline 7 & 75,7 & 74,5 & 76,8 & 4,9 & 6,97 \\
\hline 8 & 79,8 & 78,7 & 80,9 & 4,1 & 5,46 \\
\hline 9 & 83,3 & 82,1 & 84,4 & 3,5 & 4,35 \\
\hline 10 & 86,2 & 85,0 & 87,3 & 2,9 & 3,49 \\
\hline 11 & 88,6 & 87,5 & 89,7 & 2,4 & 2,83 \\
\hline 12 & 90,7 & 89,5 & 91,8 & 2,0 & 2,31 \\
\hline 13 & 92,4 & 91,3 & 93,5 & 1,7 & 1,89 \\
\hline 14 & 93,8 & 92,7 & 94,9 & 1,4 & 1,56 \\
\hline
\end{tabular}




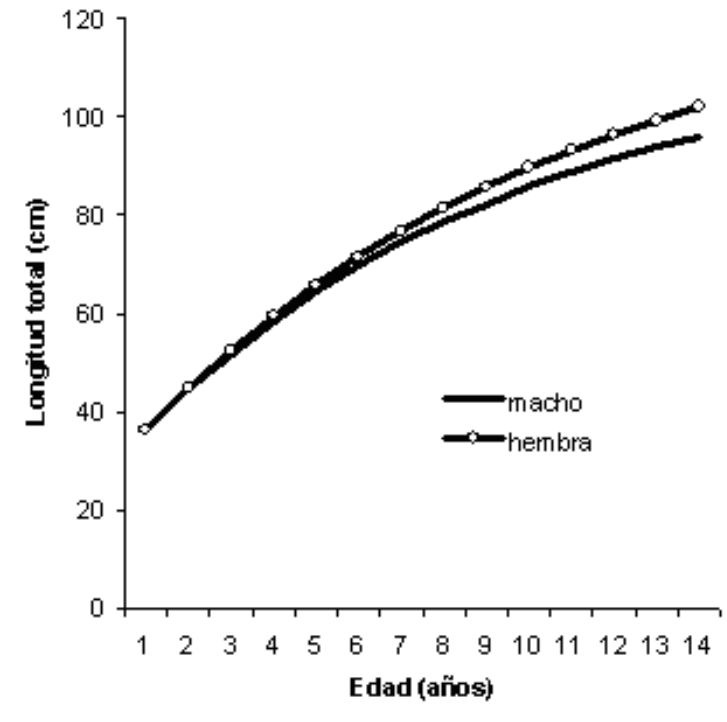

Figura 6

Curva de crecimiento en longitud determinada por ajuste no lineal para longitud-edad actual, en machos y hembras de $M$. magellanicus

Growth curve in length determined by non linear fit for present age length in males and females of M. magellanicus

\section{Crecimiento en peso}

\section{Relación entre longitud y peso}

Se estableció la relación existente entre la longitud total (LT) y el peso total de merluza de cola, por sexo. Esta relación quedó representada por las siguientes funciones potenciales (Fig. 7):

Hembras: $W=3,536 \cdot 10^{-3} * L T^{2,965}$

Machos: $W=4,9468 \cdot 10^{-3} * L T^{2,872}$

Combinados: $W=3,383 \cdot 10^{-3} * L T^{2,961}$

\section{Estimación de crecimiento en peso}

Las ecuaciones que representan el crecimiento en peso (Fig. 8) son las siguientes:

Hembras: $W_{t}=3305,85 g\left[1-e^{-0,16704(t+0,81273)}\right]^{2,965}$

Machos: $W_{t}=2379,42 g\left[1-e^{-0,19127(t+0,78882)}\right]^{2,872}$

Combinados: $W_{t}=2.931,22 g\left[1-e^{-0,17588(t+0,81239)}\right]^{2,96}$

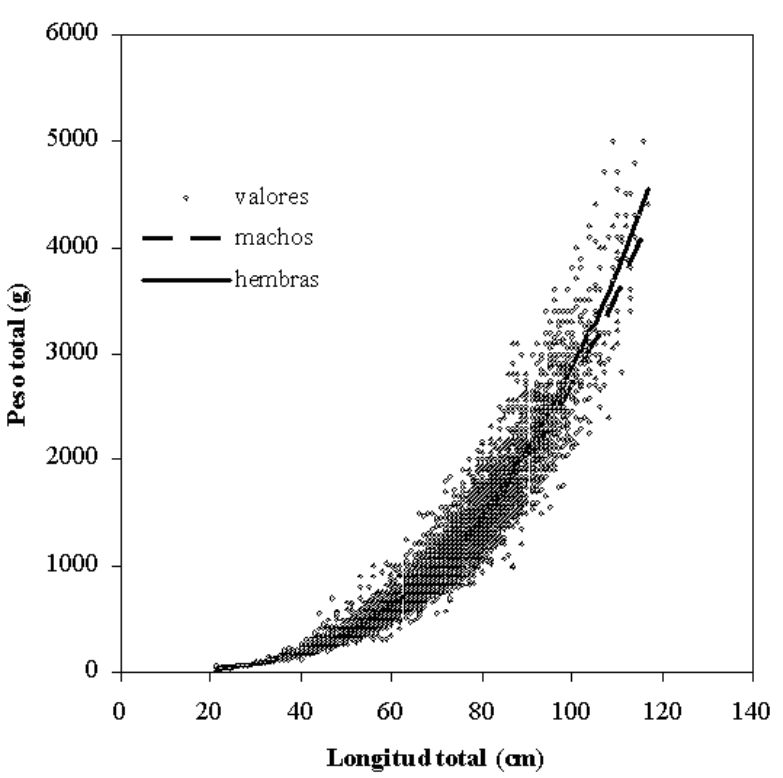

Figura 7

Relación entre la longitud total y el peso total para machos y hembras de $M$. magellanicus

Relationship between total length and total weight for males and females of $M$. magellanicus

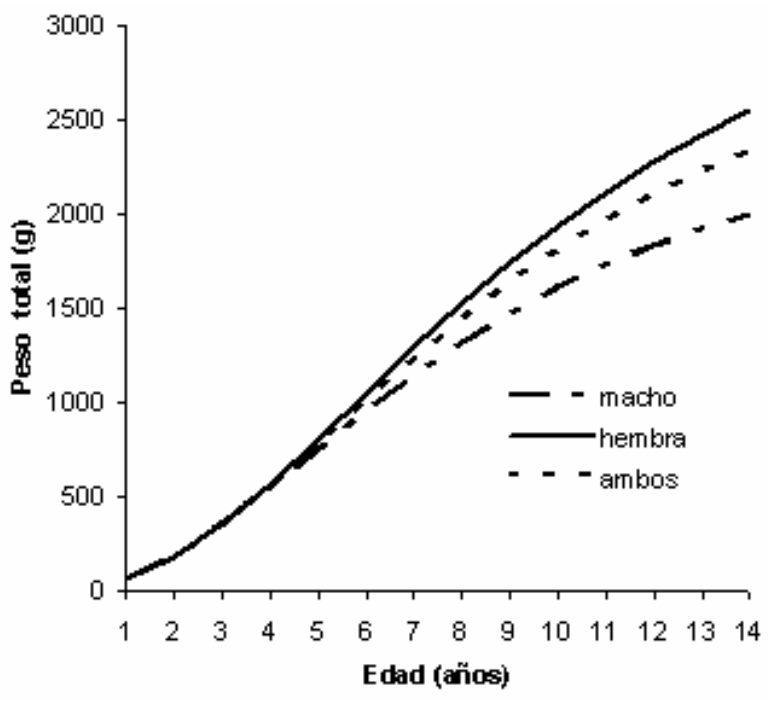

Figura 8

Curvas de crecimiento en peso para machos, hembras y para la combinación de ambos de $M$. magellanicus

Growth curves in weight for males, females and for both sexes combined of $M$. magellanicus 
Tabla 10

Longitudes promedios observadas a la edad actual y valores de estadísticos asociados para machos de M. magellanicus

Mean lengths observed at age and associated statistical values for males of M. magellanicus

\begin{tabular}{|c|c|c|c|c|c|c|c|c|}
\hline \multirow{2}{*}{$\begin{array}{l}\text { Edad } \\
\text { (años) }\end{array}$} & \multirow{2}{*}{$\begin{array}{l}\text { Long. prom. } \\
\text { edad actual } \\
(\mathrm{cm})\end{array}$} & \multirow{2}{*}{$\begin{array}{c}\text { Error } \\
\text { estándar }\end{array}$} & \multirow{2}{*}{$\begin{array}{c}\mathrm{N}^{\circ} \\
\text { anillos } \\
\text { medidos }\end{array}$} & \multicolumn{2}{|c|}{ Intervalos $(95 \%)$} & \multirow{2}{*}{$\begin{array}{l}\text { Long. estim. } \\
\text { (cm) }\end{array}$} & \multirow{2}{*}{$\begin{array}{c}\text { Tasa } \\
\text { incr. abs. } \\
(\mathrm{cm})\end{array}$} & \multirow{2}{*}{$\begin{array}{c}\text { Tasa } \\
\text { incr. rel. } \\
(\%) \\
\end{array}$} \\
\hline & & & & $\begin{array}{c}\text { Inferior } \\
(\mathrm{cm})\end{array}$ & $\begin{array}{c}\text { Superior } \\
(\mathrm{cm})\end{array}$ & & & \\
\hline 1 & 36,1 & 4,1 & 36 & 30 & 48 & 36,37 & 36,37 & \\
\hline 2 & 44,2 & 6,0 & 193 & 30 & 60 & 44,58 & 8,21 & 22,56 \\
\hline 3 & 51,7 & 6,9 & 318 & 25 & 83 & 51,92 & 7,34 & 16,48 \\
\hline 4 & 58,6 & 7,3 & 475 & 26 & 84 & 58,49 & 6,57 & 12,66 \\
\hline 5 & 64,1 & 7,9 & 479 & 32 & 88 & 64,38 & 5,88 & 10,06 \\
\hline 6 & 70,3 & 7,7 & 512 & 38 & 98 & 69,64 & 5,26 & 8,18 \\
\hline 7 & 74,2 & 7,4 & 425 & 34 & 94 & 74,35 & 4,71 & 6,76 \\
\hline 8 & 78,1 & 7,5 & 78 & 58 & 99 & 78,57 & 4,22 & 5,67 \\
\hline 9 & 81,4 & 8,6 & 224 & 63 & 104 & 82,34 & 3,77 & 4,8 \\
\hline 10 & 84,2 & 9,2 & 148 & 65 & 106 & 85,72 & 3,38 & 4,1 \\
\hline 11 & 91,8 & 7,5 & 49 & 77 & 110 & 88,74 & 3,02 & 3,53 \\
\hline 12 & 92,2 & 7,5 & 31 & 77 & 104 & 91,44 & 2,70 & 3,05 \\
\hline 13 & 97,0 & 6,7 & 6 & 86 & 105 & 93,86 & 2,42 & 2,65 \\
\hline 14 & & & & & & 96,03 & 2,17 & 2,31 \\
\hline 15 & & & & & & 97,97 & 1,94 & 2,02 \\
\hline
\end{tabular}

Tabla 11

Longitudes promedios a la edad actual y valores de estadísticos asociados para hembras de M. magellanicus

Mean length observed at age and associated statistical values for females of M. magellanicus

\begin{tabular}{|c|c|c|c|c|c|c|c|c|}
\hline \multirow{2}{*}{$\begin{array}{l}\text { Edad } \\
\text { (años) }\end{array}$} & \multirow{2}{*}{$\begin{array}{l}\text { Long. prom. } \\
\text { edad actual } \\
(\mathrm{cm})\end{array}$} & \multirow{2}{*}{$\begin{array}{c}\text { Error } \\
\text { estándar }\end{array}$} & \multirow{2}{*}{$\begin{array}{c}\mathrm{N}^{\circ} \\
\text { ejemplares }\end{array}$} & \multicolumn{2}{|c|}{ Intervalos $(95 \%)$} & \multirow{2}{*}{$\begin{array}{l}\text { Long. } \\
\text { estimada } \\
(\mathrm{cm})\end{array}$} & \multirow{2}{*}{$\begin{array}{c}\text { Tasa } \\
\text { incr. abs. } \\
(\mathrm{cm})\end{array}$} & \multirow{2}{*}{$\begin{array}{c}\text { Tasa } \\
\text { incr. rel. } \\
(\%)\end{array}$} \\
\hline & & & & $\begin{array}{c}\text { Inferior } \\
(\mathrm{cm})\end{array}$ & $\begin{array}{c}\text { Superior } \\
(\mathrm{cm})\end{array}$ & & & \\
\hline 1 & 36,9 & 4 & 29 & 28 & 44 & 36,26 & 36,26 & \\
\hline 2 & 44,5 & 5,6 & 179 & 34 & 63 & 44,74 & 8,48 & 23,38 \\
\hline 3 & 52 & 8 & 315 & 31 & 75 & 52,43 & 7,69 & 17,2 \\
\hline 4 & 60 & 7,7 & 469 & 42 & 80 & 59,42 & 6,98 & 13,32 \\
\hline 5 & 66 & 7,8 & 490 & 42 & 93 & 65,75 & 6,34 & 10,67 \\
\hline 6 & 72,2 & 7,7 & 422 & 51 & 103 & 71,51 & 5,75 & 8,75 \\
\hline 7 & 77,6 & 7,9 & 568 & 58 & 110 & 76,73 & 5,22 & 6,17 \\
\hline 8 & 80,5 & 8 & 544 & 54 & 107 & 81,46 & 4,74 & 6,17 \\
\hline 9 & 84,9 & 9,1 & 404 & 64 & 110 & 85,76 & 4,3 & 5,28 \\
\hline 10 & 90,9 & 9,2 & 206 & 71 & 116 & 89,66 & 3,9 & 4,55 \\
\hline 11 & 93,8 & 9,6 & 107 & 71 & 117 & 93,21 & 3,54 & 3,95 \\
\hline 12 & 98,5 & 10,6 & 45 & 77 & 114 & 96,42 & 3,21 & 3,45 \\
\hline 13 & 100,6 & 6,6 & 16 & 92 & 115 & 99,34 & 2,92 & 3,03 \\
\hline 14 & 103,1 & 7,9 & 8 & 93 & 113 & 101,98 & 2,65 & 2,66 \\
\hline 15 & & & & & & 104,39 & 2,4 & 2,36 \\
\hline
\end{tabular}


Tabla 12

Valores de los parámetros de crecimiento en longitud a la edad actual de M. magellanicus, estimados mediante ajuste no lineal de la función de crecimiento de von Bertalanffy

Values of the length growth parameters at age of M. magellanicus estimated by nonlinear regression of the von Bertalanffy growth equation

\begin{tabular}{|c|c|c|c|c|c|}
\hline \multirow[t]{2}{*}{ Sexo } & \multirow[t]{2}{*}{ Parámetros } & \multirow{2}{*}{$\begin{array}{c}\text { Valor } \\
\text { estimado } \\
\end{array}$} & \multirow{2}{*}{$\begin{array}{c}\text { Error } \\
\text { estándar }\end{array}$} & \multicolumn{2}{|c|}{ Intervalos (95\%) } \\
\hline & & & & Inferior & Superior \\
\hline \multirow{3}{*}{ Machos } & Loo & 111,87 & 3,05543 & 105,88 & 117,86 \\
\hline & $\mathrm{K}$ & 0,11687 & 0,0081 & 0,10099 & 0,13275 \\
\hline & $\mathrm{t}_{0}$ & $-2,32667$ & 0,18145 & $-2,68231$ & $-1,97103$ \\
\hline \multirow{3}{*}{ Hembras } & Loo & 127,97 & 4,04027 & 120,05 & 135,89 \\
\hline & $\mathrm{K}$ & 0,09733 & 0,00532 & 0,08357 & 0,11109 \\
\hline & $\mathrm{t}_{0}$ & $-2,43477$ & 0,13976 & $-2,78103$ & $-2,23317$ \\
\hline \multirow{3}{*}{ Ambos } & Loo & 124,4 & 2,92222 & 118,67 & 130,13 \\
\hline & $\mathrm{K}$ & 0,09872 & 0,00532 & 0,08829 & 0,10915 \\
\hline & $\mathrm{t}_{0}$ & $-2,5071$ & 0,13976 & $-2,78103$ & $-2,23317$ \\
\hline
\end{tabular}

Los valores de los parámetros y sus intervalos de confianza al $95 \%$ se presentan en la Tabla 13 . Por otra parte, los pesos promedio estimados a la edad y las tasas de crecimiento correspondientes por sexo, se entregan en las Tablas 14 y 15, donde se observa que los valores calculados, son muy similares hasta la edad 4 y desde la edad 5 se observan las diferencias propias de pesos entre sexos, alcanzando las hembras tallas mayores a cada edad. También se observa que mientras las hembras alcanzan una tasa de incremento absoluta máxima a la edad de 6 años, en los machos es a los 5 años, lo que podría estar relacionado con una madurez sexual más temprana de éstos.

\section{Mortalidad natural}

\section{Estimación por métodos bioanalógicos}

Los valores de la tasa instantánea de mortalidad natural (M) estimados por los distintos métodos bioanalógicos se presentan en la Tabla 16.

Los valores promedios de $\mathrm{M}$, obtenidos por los métodos fluctuaron entre 0,19 y 0,32 para machos y entre 0,17 y 0,28 para las hembras, señalando que la varianza asociada al método de Pauly en ambos sexos fue la menor, modelo que consideró una temperatura promedio anual de su hábitat de $7^{\circ} \mathrm{C}$. De esta forma, el valor de mortalidad natural ponderado por el inverso de la varianza alcanzó para los machos un valor de $\mathrm{M}=$ 0,2668 y para las hembras un $\mathrm{M}=0,2314$ (Tabla 16).

\section{Estimación de $M$ por métodos de curva de captura}

Las ecuaciones de regresión ajustadas para ambos sexos fueron:

Machos: $\mathrm{LnC}\left(\mathrm{L} 1, \mathrm{~L}_{\infty}\right)=7,9116+3,8882 \ln \left(\mathrm{L}_{\infty}-\mathrm{L} 1\right)$

$\left(\mathrm{R}^{2}=0,961\right)$

Hembras: $\operatorname{LnC}\left(\mathrm{L} 1, \mathrm{~L}_{\infty}\right)=7,3912+3,6208 \ln \left(\mathrm{L}_{\infty}-\mathrm{L} 1\right)$ $\left(\mathrm{R}^{2}=0,954\right)$

Por otra parte, la curva de captura basada en composición por edades se estructuró sobre la base de la composición de edades del año 1972. La parte descendente de la curva permitió ajustar una regresión lineal cuya pendiente corresponde a una estimación de $\mathrm{M}$, al no existir prácticamente mortalidad por pesca. Las ecuaciones de regresión para machos y hembras son las siguientes:

Machos: $\operatorname{LnC}\left(\mathrm{t}_{1}, \mathrm{t}_{2}\right)=9,30015-0,6620 * \mathrm{t}\left(\mathrm{R}^{2}=0,970\right)$

Hembras: $\operatorname{LnC}\left(\mathrm{t}_{1}, \mathrm{t}_{2}\right)=8,71360-0,5588 * \mathrm{t}\left(\mathrm{R}^{2}=0,922\right)$ 
Tabla 13

Valores de los parámetros de crecimiento en peso para M. magellanicus, estimados mediante ajuste no lineal de la ecuación de crecimiento de von Bertalanffy

Values of the growth parameters in weight estimated for M. magellanicus, by nonlinear regression of the von Bertalanffy growth equation

\begin{tabular}{ccccc}
\hline Sexo & Parámetros & Valor estimado & \multicolumn{2}{c}{ Intervalos (95\%) } \\
\cline { 3 - 5 } & & & Inferior & Superior \\
\hline Machos & $\mathrm{W}_{\infty}$ & 2379,42 & 1708,43 & 3248,26 \\
& $\mathrm{~K}$ & 0,19127 & 0,18641 & 0,19613 \\
& $\mathrm{t}_{0}$ & $-0,78882$ & $-0,82228$ & $-0,75536$ \\
& & & & \\
Hembras & $\mathrm{W}_{\infty}$ & 3305,85 & 2411,03 & 4567,36 \\
& $\mathrm{~K}$ & 0,16704 & 0,16285 & 0,17123 \\
& $\mathrm{t}_{0}$ & $-0,81273$ & $-0,84566$ & $-0,77980$ \\
& & & & 3653,15 \\
& $\mathrm{~W}_{\infty}$ & 2931,22 & 2330,12 & 0,17907 \\
& $\mathrm{~K}$ & 0,17588 & 0,17269 & $-0,78820$ \\
\hline
\end{tabular}

Tabla 14

Peso promedio estimado por edad y valores de las tasas de incremento para machos de M. magellanicus

Mean weight estimated for age and growth rate values for males of M. magellanicus

\begin{tabular}{|c|c|c|c|c|c|}
\hline \multirow{2}{*}{$\begin{array}{l}\text { Edad } \\
\text { (años) }\end{array}$} & \multirow{2}{*}{$\begin{array}{l}\text { Peso estimado } \\
\text { (g) }\end{array}$} & \multicolumn{2}{|c|}{ Intervalos $(95 \%)$} & \multirow{2}{*}{$\begin{array}{c}\text { Tasa } \\
\text { incr. absoluto } \\
(\mathrm{cm})\end{array}$} & \multirow{2}{*}{$\begin{array}{c}\text { Tasa } \\
\text { incr. relativo } \\
\%\end{array}$} \\
\hline & & Inferior & Superior & & \\
\hline 1 & 67,83 & 49,83 & 90,31 & 67,83 & \\
\hline 2 & 188,23 & 135,00 & 256,94 & 120,40 & 177,50 \\
\hline 3 & 354,85 & 251,89 & 489,53 & 166,62 & 88,52 \\
\hline 4 & 548,33 & 387,39 & 760,14 & 193,48 & 54,52 \\
\hline 5 & 751,75 & 530,02 & 1044,29 & 203,42 & 37,10 \\
\hline 6 & 952,56 & 671,26 & 1323,97 & 200,82 & 26,71 \\
\hline 7 & 1142,51 & 805,38 & 1587,46 & 189,94 & 19,94 \\
\hline 8 & 1316,75 & 928,98 & 1828,06 & 174,25 & 15,25 \\
\hline 9 & 1473,05 & 1040,40 & 2042,79 & 156,30 & 11,87 \\
\hline 10 & 1610,88 & 1139,18 & 2231,17 & 137,83 & 9,36 \\
\hline 11 & 1730,85 & 1225,61 & 2394,26 & 119,97 & 7,45 \\
\hline 12 & 1834,20 & 1300,49 & 2534,00 & 103,36 & 5,97 \\
\hline 13 & 1922,54 & 1364,83 & 2652,77 & 88,33 & 4,82 \\
\hline 14 & 1997,55 & 1419,76 & 2753,08 & 75,01 & 3,90 \\
\hline
\end{tabular}


Tabla 15

Peso promedio estimado para cada edad y tasas de incremento para hembras de M. magellanicus

Mean weight estimated for age and growth increment values rate for females of M. magellanicus

\begin{tabular}{|c|c|c|c|c|c|}
\hline \multirow{2}{*}{$\begin{array}{l}\text { Edad } \\
\text { (años) }\end{array}$} & \multirow{2}{*}{$\begin{array}{l}\text { Peso estimado } \\
\text { (g) }\end{array}$} & \multicolumn{2}{|c|}{ Intervalos $(95 \%)$} & \multirow{2}{*}{$\begin{array}{c}\text { Tasa } \\
\text { incr. absoluto } \\
(\mathrm{cm}) \\
\end{array}$} & \multirow{2}{*}{$\begin{array}{c}\text { Tasa } \\
\text { incr. relativo } \\
\%\end{array}$} \\
\hline & & Inferior & Superior & & \\
\hline 1 & 61,79 & 46,12 & 83,23 & 61,79 & \\
\hline 2 & 180,28 & 131,34 & 249,05 & 118,50 & 191,78 \\
\hline 3 & 354,79 & 255,70 & 495,57 & 174,51 & 96,80 \\
\hline 4 & 569,04 & 407,95 & 799,12 & 214,25 & 60,39 \\
\hline 5 & 806,11 & 576,46 & 1134,91 & 237,06 & 41,66 \\
\hline 6 & 1051,50 & 751,25 & 1481,81 & 245,40 & 30,44 \\
\hline 7 & 1294,17 & 924,62 & 1823,80 & 242,67 & 23,08 \\
\hline 8 & 1526,36 & 1091,12 & 2149,80 & 232,19 & 17,94 \\
\hline 9 & 1743,13 & 1247,22 & 2452,89 & 216,77 & 14,20 \\
\hline 10 & 1941,74 & 1390,87 & 2729,36 & 198,61 & 11,39 \\
\hline 11 & 2121,06 & 1521,17 & 2977,83 & 179,32 & 9,24 \\
\hline 12 & 2281,10 & 1638,01 & 3198,53 & 160,04 & 7,55 \\
\hline 13 & 2422,61 & 1741,81 & 3392,75 & 141,51 & 6,20 \\
\hline 14 & 2546,80 & 1833,35 & 3562,38 & 124,19 & 5,13 \\
\hline
\end{tabular}

Tabla 16

Mortalidad natural estimada por métodos bioanalógicos para cada sexo de $M$. magellanicus

Natural mortality estimated by bioanalogical methods for each sex of M. magellanicus

\begin{tabular}{cccccc}
\hline & & \multicolumn{2}{c}{ Método bioanalógico } \\
& & Taylor & Alagaraja & Alverson \& & Pauly \\
& & $(1960)$ & $(1984)$ & Carney (1975) & $(1980)$ \\
\hline \multirow{2}{*}{ Machos } & M promedio & 0,2076 & 0,3223 & 0,2176 & 0,1898 \\
& varianza & $7,83 \mathrm{E}-06$ & $1,98 \mathrm{E}-05$ & $4,84 \mathrm{E}-06$ & $4,38 \mathrm{E}-06$ \\
\hline Hembras & M promedio & 0,1795 & 0,2787 & 0,2001 & 0,1698 \\
& varianza & $5,45 \mathrm{E}-06$ & $1,31 \mathrm{E}-05$ & $4,46 \mathrm{E}-06$ & $3,24 \mathrm{E}-06$ \\
\hline \multirow{3}{*}{ Machos } & & M ponderada & & & \\
Hembras & M ponderada & 0,2668 & & & \\
\hline
\end{tabular}

Los valores estimados de $\mathrm{M}$ con su correspondiente varianza e intervalo de confianza por sexo se presentan en la Tabla 17. Estos valores son inferiores a los obtenidos por el método de curva de captura basado en composición de talla. Además, las estimaciones de M obtenidas por los métodos bioanalógicos son muy inferiores a los calculados mediante la curva de captura.

\section{Discusión}

El examen de los otolitos sagitta ha permitido observar y discriminar con claridad los anillos hialinos y opacos de crecimiento y su depósito estacional en la forma de anillos estrechos oscuros y anchos opacos, respectivamente, lo que corrobora los resultados entregados sobre la formación de los anillos de crecimiento para esta especie por diferentes autores (Aguayo 1974, Aguayo \& Gili 1984, Tomo \& Torno 1987, Young et al. 1998, Cubillos et al. 1998). 


\section{Tabla 17}

\section{Valores de mortalidad natural con sus respectivas varianzas e intervalos de confianza (95\%) estimados para $M$. magellanicus} por diferentes métodos de curva de captura

Natural mortality values with its variances and confidence intervals (95\%), estimated for M. magellanicus by different catch curve methods

\begin{tabular}{|c|c|c|c|c|c|}
\hline \multicolumn{6}{|c|}{ Métodos de curva de captura } \\
\hline \multicolumn{6}{|c|}{ Curva de captura basada en estructura de tallas (Jones \& van Zalinge 1982) } \\
\hline & Mortalidad & Varianza & \multicolumn{2}{|c|}{ Intervalo de confianza $(95 \%)$} & $\mathrm{R}^{2}$ \\
\hline Machos & 0,722 & 0,10232 & 0,577 & 0,868 & 0,961 \\
\hline Hembras & 0,590 & 0,09055 & 0,474 & 0,563 & 0,953 \\
\hline \multicolumn{6}{|c|}{ Curva de captura basada en estructura de edades (Chapman \& Robson 1960) } \\
\hline & Mortalidad & Varianza & \multicolumn{2}{|c|}{ Intervalo de confianza $(95 \%)$} & $\mathrm{R}^{2}$ \\
\hline Machos & 0,622 & 0,00269 & 0,529 & 0,795 & 0,964 \\
\hline Hembras & 0,559 & 0,10659 & 0,333 & 0,784 & 0,920 \\
\hline
\end{tabular}

En particular, los resultados obtenidos en el presente trabajo señalan la formación de un anillo hialino (annulus) y uno opaco cada año con una mayor formación del borde hialino en invierno, entre junio y agosto. Esto, en gran medida, es coincidente con lo registrado para esta especie frente a las costas del Océano Pacífico suroriental (Aguayo 1974, Aguayo \& Gili 1984, Cubillos et al. 1998), así como en el Océano Atlántico suroccidental (Tomo \& Torno 1987, Chesheva 1996, Giussi 1996).

Uno de los aspectos básicos que más se ha tenido en cuenta en este estudio es el nivel de exactitud en la asignación de edades, ya que influye en los estudios de dinámica poblacional. Existen muchos ejemplos en la que errores en la asignación de edad han contribuído a la sobrexplotación de una población o especie, asociado a menudo con una subestimación de la edad (Campana 2001). Generalmente, este tipo de problema se ha traducido en un excesivo estimado de tasas de crecimiento y mortalidad, como es el caso del "orange roughy" (Hoplostethus atlanticus Collett) que fue intensamente capturado hasta casi el colapso de la población, sobre la base de una longevidad presunta de 20-30 años y para el que actualmente se postula una longevidad cercana a los 100 años (Smith et al. 1995).

Hay que destacar que en el presente estudio se utilizó la lectura de anillos anuales en los otolitos sagitales, estructura usada en todos los trabajos publicados hasta la fecha en M. magellanicus (Aguayo 1974, Aguayo \& Gili 1984, Tomo \& Torno 1987, Young et al. 1998, Cubillos et al. 1998) así como también, la técnica de retrocálculo (Francis 1990,
Young et al. 1998) y la estimación de longitud a edad actual (Young et al. 1998, Cubillos et al. 1998).

Las primeras estimaciones de crecimiento realizadas por Aguayo (1974) para M. magellanicus presente en aguas chilenas, fueron sin diferenciar sexo y para un rango de talla entre 24 y $73 \mathrm{~cm}$ de longitud total. Los ajustes realizados por el autor mediante el método de Allen (1966), permitieron estimar una longitud asintótica de $66,8 \mathrm{~cm}$ (Tabla 18). Este bajo valor de $\mathrm{L}_{\infty}$ se explicaría por el menor rango de longitudes analizadas disponibles, esto es, con una menor presencia de tallas adultas.

Aguayo \& Gili (1984) efectuaron un nuevo estudio de crecimiento sobre la base de lectura de 2.900 otolitos recolectados en las capturas de barcos arrastreros fábrica entre los paralelos $43^{\circ} 00^{\prime} \mathrm{S}$ y $57^{\circ} 00^{\prime} \mathrm{S}$. El número de edades determinadas fue de 13 años para un rango de talla entre 28 y $114 \mathrm{~cm}$ y no detectaron diferencias significativas entre machos $\mathrm{y}$ hembras en la comparación de la relación entre la longitud del pez y el radio del otolito. De esta forma, los parámetros de la función de crecimiento en longitud, incluyendo ambos sexos, ajustados por estos autores, por el método de Allen, entregó valores de $\mathrm{L}_{\infty}$ $=103 \mathrm{~cm} \mathrm{LT}, \mathrm{K}=0,2171$ y $_{\mathrm{o}}=0,21891$ (Tabla 18).

Young et al. (1998) realizaron un estudio con una muestra de otolitos sagitales de ejemplares de merluza de cola recolectados el año 1996 en un rango de tallas entre 34 y $111 \mathrm{~cm}$ de longitud total, observando diferencias significativas en el crecimiento entre sexos. Las edades determinadas fueron de 2 a 15 años en las 


\section{Tabla 18}

Valores de los parámetros de crecimiento para M. magellanicus estimados por diferentes autores para el Océano Pacífico suroriental y el Océano Atlántico suroccidental

Growth parameters values for M. magellanicus estimated for differentes authors for Southeastern Pacific Ocean and the Southwestern Atlantic

\begin{tabular}{|c|c|c|c|c|c|c|c|}
\hline \multirow[t]{2}{*}{ Sexo } & \multicolumn{3}{|c|}{ Parámetros } & \multirow{2}{*}{$\begin{array}{c}\text { Estructura } \\
\text { medida }\end{array}$} & \multirow{2}{*}{$\begin{array}{c}\text { Método } \\
\text { determinación edad }\end{array}$} & \multirow{2}{*}{$\begin{array}{l}\text { Datos } \\
\text { ajuste }\end{array}$} & \multirow{2}{*}{$\begin{array}{c}\text { Fuente } \\
\text { autor }\end{array}$} \\
\hline & $\mathbf{L}_{\infty}$ & $\mathbf{K}$ & $t_{0}$ & & & & \\
\hline \multicolumn{8}{|c|}{ Océano Pacífico } \\
\hline $\mathrm{H}+\mathrm{M}$ & 66,8 & 0,3598 & $-0,071$ & Long. anillo & Regresión & L. prom-edad & 1 \\
\hline $\mathrm{H}+\mathrm{M}$ & 103,2 & 0,2171 & 0,2189 & Long. anillo & Regresión & L. prom-edad & 2 \\
\hline M & 115,9 & 0,1118 & $-1,808$ & $\mathrm{~N}^{\circ}$ anillos & E. actual & L. individual & 3 \\
\hline $\mathrm{H}$ & 123,3 & 0,1173 & $-1,431$ & $\mathrm{~N}^{\circ}$ anillos & E. actual & L. individual & 3 \\
\hline M & 100,6 & 0,1653 & $-0,788$ & Radio anillo & Retrocálculo & L. individual & 3 \\
\hline $\mathrm{H}$ & 101,5 & 0,1794 & $-0,765$ & Radio anillo & Retrocálculo & L. individual & 3 \\
\hline M & 117,7 & 0,1113 & $-1,674$ & $\mathrm{~N}^{\circ}$ anillos & E. actual & L. individual & 4 \\
\hline $\mathrm{H}$ & 129,9 & 0,1134 & $-0,997$ & $\mathrm{~N}^{\circ}$ anillos & E. actual & L. individual & 4 \\
\hline M & 95,6 & 0,2110 & $-0,349$ & Long. anillo & Retrocálculo & L. individual & 4 \\
\hline $\mathrm{H}$ & 104,2 & 0,1894 & $-0,279$ & Long. anillo & Retrocálculo & L. individual & 4 \\
\hline $\mathrm{M}+\mathrm{H}$ & 83,1 & 0,146 & $-0,809$ & Radio anillo & Peso otolitos & Frec. tallas & 5 \\
\hline $\mathrm{M}+\mathrm{H}$ & 87,2 & 0,1420 & $-0,690$ & Radio anillo & E. actual & Edad - W.otol & 5 \\
\hline M & 95,65 & 0,1913 & $-0,7888$ & Radio anillo & Retrocálculo & L. individual & 6 \\
\hline $\mathrm{H}$ & 105,48 & 0,1670 & $-0,8127$ & Radio anillo & Retrocálculo & L. individual & 6 \\
\hline $\mathrm{M}+\mathrm{H}$ & 101,29 & 0,1759 & $-0,8124$ & Radio anillo & Retrocálculo & L. individual & 6 \\
\hline M & 111,87 & 0,1169 & $-2,3267$ & $\mathrm{~N}^{\circ}$ anillos & E. actual & L. individual & 6 \\
\hline $\mathrm{H}$ & 127,97 & 0,0973 & $-2,4348$ & $\mathrm{~N}^{\circ}$ anillos & E. actual & L. individual & 6 \\
\hline \multicolumn{8}{|c|}{ Océano Atlántico } \\
\hline$M^{*}$ & 47,8 & 0,0949 & $-2,550$ & Secciones & E. actual & L. prom.-edad & 7 \\
\hline $\mathrm{H}^{*}$ & 49,0 & 0,0867 & $-3,060$ & Secciones & E. actual & L. prom.-edad & 7 \\
\hline $\mathrm{M}+\mathrm{H}$ & 88,5 & 0,2292 & $-0,971$ & $\mathrm{~N}^{\circ}$ anillos & E. actual & L. prom.-edad & 8 \\
\hline \multicolumn{8}{|c|}{ M. novaezelandiae } \\
\hline M & 90,7 & 0,2560 & $-1,210$ & $\mathrm{~N}^{\circ}$ anillos & E. actual & L. individual & 9 \\
\hline $\mathrm{H}$ & 99,3 & 0,2030 & $-1,480$ & $\mathrm{~N}^{\circ}$ anillos & E. actual & L. individual & 9 \\
\hline $\mathrm{M}+\mathrm{H}$ & 95,6 & 0,2260 & $-1,220$ & $\mathrm{~N}^{\circ}$ anillos & E. actual & L. individual & 9 \\
\hline \multicolumn{4}{|c|}{ M: macho; H: hembra } & \multicolumn{4}{|c|}{$*$ L. preanal: 47,8 = 109,6 cm LT; 49,0 = 117,2 LT } \\
\hline \multicolumn{8}{|c|}{$\begin{array}{l}\text { 1. Aguayo (1974), 2. Aguayo \& Gili (1984), 3. Young et al. (1998), 4. Young et al. (1998**), 5. Cubillos et al. (1998), } 6 \\
\text { Este estudio, 7. Tomo \& Torno (1987), 8. Chesheva (1996), 9. Kenchington \& Augustine (1987). } \\
(* * \text { data Aguayo \& Gili 1984) }\end{array}$} \\
\hline
\end{tabular}


hembras y de 2 a 14 años en los machos. Los parámetros fueron estimados por sexo mediante regresiones no lineales (Tabla 18), considerando valores de longitud total a la edad actual y retrocalculadas.

La aplicación de pruebas estadísticas como el $\mathrm{T}^{2}$ de Hotelling y el de Chen et al. (1992), confirman la existencia de diferencias significativas en crecimiento de machos y hembras ya observada por Young et al. (1998) y Ojeda et al. (1998).

Los valores de los parámetros obtenidos con información de longitud-edad a la edad actual muestran con claridad valores de $\mathrm{L}_{\infty}$ superiores a los ajustados con longitudes retrocalculadas, pero con valores menores de $\mathrm{K}$ y de $\mathrm{t}_{\mathrm{o}}$ (Tabla 15), lo que se explica por la correlación inversa que existe entre $\mathrm{L}_{\infty} \mathrm{y} \mathrm{K}$.

En el mismo trabajo, estos autores (Young et al. 1998, Ojeda et al. 1998) analizan los datos de lecturas correspondiente a la muestra recolectada el año 1982 por Aguayo \& Gili (1984), pero analizada con la nueva técnica indicada por Francis (1990). Los resultados obtenidos para cada sexo muestran valores distintos, los que al ser comparados establecen que las hembras presentan crecimientos significativamente diferentes a los machos, alcanzado una longitud asintótica promedio mayor (Tabla 18).

Young et al. (1998) señalan que el procedimiento de retrocálculo subestimaría la longitud asintótica, dado que en las capturas comerciales se observan ejemplares mayores de $110 \mathrm{~cm}$ de longitud total. Por esta razón, consideran que las curvas que mejor representan el crecimiento en longitud de merluza de cola son las ajustadas con la información de "longitud-edad actual". Esta afirmación podría ser válida si en la muestra estuviera representada toda la estructura de tallas de la población, pero dado que la fracción de tallas más jóvenes está poco representada ( $<3$ años), ya sea por la selectividad del arte de arrastre (malla de $130 \mathrm{~mm}$ ) o una distribución espacial diferente al área de captura, $\mathrm{L}_{\infty}$ estaría sobreestimada. El hecho que en la captura se hayan observado algunos ejemplares de $110 \mathrm{~cm}$ LT o mayores no condiciona el $\mathrm{L}_{\infty}$, ya que de acuerdo con el modelo, éste no representa la mayor longitud observada sino un promedio, pudiendo estar las mayores longitudes observadas en el intervalo de confianza de esta longitud asintótica. En definitiva, se considera que la mejor ecuación que representa el crecimiento en merluza de cola es aquella determinada mediante la longitud-edad retrocalculada corregida obtenida en el presente trabajo.

En la zona centro-sur de Chile, Cubillos et al. (1998) realizaron un estudio de edad y crecimiento en merluza de cola, basándose en información de muestreos de frecuencia de tallas en las capturas de la flota cerquera, correspondientes al período 1992-1997. La metodología empleada para la determinación de edad consistió en la lectura de anillos de crecimiento, y utilización de pesos de otolitos correspondiente a muestras del año 1997, así como análisis de progresión modal correspondiente a la distribución de frecuencia de tallas mediante el programa MULTIFAN (Fournier et al. 1990 fide Cubillos et al. 1998). Los resultados obtenidos por estos autores determinaron 9 clases de edad sin separar sexos y los valores de los parámetros de crecimiento correspondieron a $\mathrm{L}_{\infty}=90,2 \mathrm{~cm} \mathrm{LT}, \mathrm{K}$ $=0,136 \mathrm{año}^{-1}$. Paralelamente, los valores de los parámetros de crecimiento estimados mediante la relación edad-peso del otolito entregaron valores levemente inferiores de $\mathrm{L}_{\infty}=87,2 \mathrm{~cm} \mathrm{LT} ; \mathrm{K}=0,142 \mathrm{y}$ $\mathrm{t}_{\mathrm{o}}=-0,690$. Ambos resultados muestran valores de $\mathrm{L}_{\infty} \mathrm{y}$ $\mathrm{K}$ inferiores a los obtenidos en el presente trabajo, lo que con seguridad está asociado al menor rango de talla examinado y a los métodos diferentes utilizados en los ajustes de crecimiento.

Por otra parte, la gran limitación que tienen los resultados obtenidos por Cubillos et al. (1998) es que la composición por tallas de las capturas de cerco (pesca pelágica) en la zona centro-sur muestra una presencia mayoritaria de ejemplares juveniles; esto es, con un sesgo importante en las tallas adultas, ya que el stock adulto se localiza principalmente entre la X y la XII Regiones (Aguayo et al. 1990, Young et al. 1998). Además, se agrega el hecho que no se efectuó el análisis en forma individual por sexos, pues como se demostró en el presente trabajo y por Young et al. (1998), las hembras presentan un crecimiento significativamente mayor que los machos a partir de la edad 4.

Uno de los primeros antecedentes sobre edad y crecimiento para $M$. magellanicus en el Océano Atlántico suroeste es el aportado por Tomo \& Torno (1987), quienes aplicando el método de lectura de anillos en los otolitos determinan la función de crecimiento para cada sexo de merluza de cola, pero no comparan el crecimiento entre hembras y machos, estableciéndolo en definitiva en función de la longitud 
preanal (Tabla 18). Las longitudes preanales asintóticas estimadas por estos autores corresponderían a longitudes totales de 117,2 y 109,6 cm para hembras y machos, respectivamente (Young et al. 1998).

Posteriormente, Chesheva (1996), con muestras obtenidas frente a las costas argentinas en 1989, determina edades de 2 a 9 años para ejemplares de merluza de cola entre 34 y $89 \mathrm{~cm} \mathrm{LT}$, sin diferenciar sexo, estimando valores de $\mathrm{L}_{\infty}=88,49 \mathrm{~cm} ; \mathrm{K}=0,2292$ $\mathrm{y}_{\mathrm{o}}=-0,9707$ (Tabla 18). Establece a la vez la relación longitud-peso con un valor de $b=2,9837$. Estos resultados hay que considerarlos en su contexto, pues al igual que los obtenidos por Cubillos et al. (1998), en dicho trabajo hay ausencia tanto de ejemplares juveniles como de los más adultos, lo que influye en la estimación de la longitud asintótica.

En aguas de Australia se encuentra Macruronus novaezelandiae (Hector, 1871), donde se han registrado ejemplares de 25 años de edad en hembras y 20 años en machos (Kenchington \& Augustine 1987). Estos autores han establecido las funciones de crecimiento por sexo, determinando un crecimiento significativamente mayor de las hembras con respecto a los machos, informando $L_{\infty}=99,3 \mathrm{~cm}$ y $L_{\infty}=90,7 \mathrm{~cm}$, respectivamente. Los valores de los parámetros de la función de crecimiento obtenidos por estos autores son muy cercanos a los estimados en el presente trabajo (Tabla 18), lo que, en gran medida, estaría indicando que el patrón de crecimiento de ambas especies se ha mantenido independiente de la influencia del ambiente, pero sí dependiente del acervo genético de ellas.

Las curvas de crecimiento en peso del modelo de von Bertalanffy establecidas en el presente estudio, para cada sexo, constituyen las primeras estimaciones realizadas en $M$. magellanicus para aguas del Océano Pacífico suroriental, con excepción de aquella efectuadas por Aguayo \& Gili (1984) sin diferenciar sexos. Los parámetros obtenidos para machos $\left(\mathrm{W}_{\mathrm{oo}}=\right.$ $2.379,42 \mathrm{~g})$ y hembras $\left(\mathrm{W}_{\infty}=3.305,85 \mathrm{~g}\right)$ muestran un crecimiento significativamente diferente entre los sexos, lo cual comienza a evidenciarse a partir de la edad 4 en favor de las hembras; ello es consistente con lo determinado mediante la curva de crecimiento en longitud, y que en gran medida, se corresponde con la talla de primera madurez sexual estimada para esta especie. Desafortunadamente, no se tienen antecedentes publicados sobre esta función, para esta especie presente en el Océano Atlántico suroccidental.
En Chile, M. magellanicus forma parte de un conjunto de peces que en la misma región, son objeto de la pesquería demersal sur-austral y, por lo tanto, están influenciados por las mismas condiciones ambientales. Los valores del coeficiente de crecimiento $\mathrm{K}$ obtenido de 0,193 y 0,167 están cercanos a los informados para otras especies que cohabitan con merluza de cola, como Genypterus blacodes (Schneider) y Seriolella caerulea Guichenot, con valores de $\mathrm{K}$ entre 0,31 y 0,43 y entre 0,25 y 0,26 , respectivamente (Chong \& Aguayo 1990, 1994), pero a la vez superiores a los de Merluccius australis (Hutton) con K entre 0,083 y 0,096 (Ojeda \& Aguayo 1986). No obstante, los actuales valores de $\mathrm{K}$ están comprendidos dentro del rango de 0,10 a 0,35 informado para poblaciones de "cod", "haddock", "pollock" (Gadidae) y merluzas (Merluciidae) (Pauly 1980).

Respecto a los valores de mortalidad natural estimados por los diferentes métodos propuestos, cabe señalar que las estimaciones por métodos bioanalógicos pueden estar subestimados y lo más probable es que los valores reales de $\mathrm{M}$ estén más próximos a los estimados con el análisis de curva de captura ( 0,7 a 0,6 en machos y 0,55 a 0,59 en hembras), tomando en cuenta que la información que se utilizó corresponde al año 1972, prácticamente cinco años antes de que se iniciará la pesquería demersal sur austral, orientada principalmente a la merluza del sur o austral (Merluccius australis), de la cual la merluza de cola es una especie incidental y una de sus principales presas. En este contexto, la mortalidad total estimada, prácticamente corresponde a mortalidad natural al estar ausente o con valores ínfimos la mortalidad por pesca.

\section{Agradecimientos}

Los autores desean expresar sus agradecimientos al Fondo de Investigación Pesquera, por autorizar la utilización de los datos obtenidos a través del proyecto FIP 97-15 con la dirección y participación los autores. Los autores desean hacer extensivos sus agradecimientos a tres revisores anónimos que con sus observaciones y sugerencias han enriquecido la presente publicación.

\section{Literatura citada}

Aguayo M. 1974. Estudio de la edad y el crecimiento de la merluza de cola (Macruronus magellanicus, Lönnberg 1907). Serie Investigación Pesquera. Instituto de Fomento Pesquero, Chile 19: 1-43. 
Aguayo M \& R Gili. 1984. Edad y crecimiento de merluza de cola (Macruronus magellanicus, Lönnberg) (Gadiformes-Gadidae). Investigación Pesquera 31: 47-57.

Aguayo M, Z Young, R Bustos, V Ojeda, T Peñailillo, R Gili, C Vera \& H Robotham. 1986. Diagnóstico de las Principales Pesquerías Nacionales Demersales (peces) Zona Sur-austral. 1985. Estado de situación del recurso. Instituto de Fomento Pesquero AP 86/55: 1-143.

Aguayo M, Z Young, R Bustos, T Peñailillo, V Ojeda, C Vera, H Hidalgo \& I Céspedes. 1987. Diagnóstico de las Principales Pesquería Nacionales Demersales (peces). Zona Sur-austral. 1986. Estado de Situación del Recurso. Instituto de Fomento Pesquero AP 87/3: 1-209.

Aguayo M, I Payá, R Bustos, V Ojeda, R Gili, C Vera, I Céspedes \& L Cid. 1990. Diagnóstico de las Principales Pesquerías Nacionales Demersales (peces) Zona Suraustral. 1988. Estado de Situación del Recurso. Instituto de Fomento Pesquero AP 89/17: 1-161.

Alagaraja K. 1984. Simple method for estimation of parameters for assessing exploited fish stocks. Indian Journal of Fisheries 31: 177-208.

Allen K. 1966. A method of fitting growth curves of the von Bertalanffy type to observed data. Journal of the Fisheries Research Board of Canada 23(3): 163-179.

Alverson DL \& MJ Carney. 1975. A graphic review of the growth and decay of population cohorts. Journal du Conseil 36: 133-143.

Angelescu V, F Gneri \& A Nani. 1958. La merluza del mar argentino (Biología y Taxonomía). Publicaciones. Servicio Hidrográfico Naval, Secretaria de Marina, Buenos Aires H 1004: 1-224.

Arana P. 1970. Nota sobre la presencia de ejemplares de merluza de cola (Macruronus magellanicus Lönnberg) frente a la costa de Valparaíso. Investigaciones Marinas 1 (3): 50-60.

Arancibia H, R Alarcón, L Cubillos, S Núñez, I Toledo, L Vilugrón, C Veloso \& H Peña. 1994. Evaluación indirecta de merluza de cola en la VIII Región. Informes Técnicos FIP -IT/93 - 09: 1-115.

Avilés S, M Aguayo, F Hinostroza \& J Cañón. 1979. Merluza de cola, En: Estado Actual de las Principales Pesquerías Nacionales. Bases para un Desarrollo Pesquero. Peces. CORFO/IFOP, AP79-18: 1-25.

Bernard R. 1981. Multivariate analysis as a mean of comparing growth in fish. Canadian Journal of Fisheries and Aquatic Science 38: 233-236.

Beverton R \& S Holt. 1957. On the dynamic of exploited fish populations. Fishery Investigations, Series 2 (19): 1533.
Campana SE. 2001. Review Paper: Accuracy, precision and quality control in age determination, including a review of the use and abuse of age validation methods. Journal of Fish Biology 59: 197-242.

Cerrato RM. 1990. Interpretable statistical test for growth comparisons using parameters in the von Bertalanffy equation. Canadian Journal of Fisheries and Aquatic Science 47: 1416-1426.

Chapman D \& D Robson. 1960. The analysis of a catch curve. Biometrics 16 (3): 354-368.

Cohen D, T Inada, T Iwamoto \& N Scialabba. 1990. Gadiform fishes of the world (Order Gadiformes). FAO Species Catalogue. FAO Fisheries Synopsis 125 (10): 1442.

Cubillos L, A Hernández, L Vilugrón, L Miranda, R Alarcón, C Pino, A Sepúlveda \& G Vásquez. 1998. Estudio biológico-pesquero de merluza de cola en el área de distribución de la pesquería pelágica centro-sur. Informe Final Proyecto FIP N 96-19: 1-158.

Csirke J 1980. Introducción a la dinámica de poblaciones de peces. FAO Documento Técnico de Pesca 192: 1-82.

Chen Y, A Jackson \& H Harvey. 1992. A comparison of von Bertalanffy and polynomial functions in modelling fish growth data. Canadian Journal of Fisheries and Aquatic Sciences 49: 1228-1235.

Chesheva ZA. 1992. Data on the biology of the Magellan hake, Macruronus magellanicus, from the Southwestern Atlantic. Journal of Ichthyology 32(7): 137-141.

Chesheva ZA. 1996. The determination of the age and the estimation of the growth rate for the Magellan hake Macruronus magellanicus of south-west Atlantic. Journal of Ichthyology 36 (1): 55-59.

Chilton ED \& RJ Beamish. 1982. Age determination methods for fishes studied by the Groundfish Program at the Pacific Biological Station. Canadian Special Publication Fisheries and Aquatic Sciences 60, 102 pp.

Chong J \& M Aguayo. 1990. Determinación de edad y estimación de los parámetros de crecimiento del congrio dorado, Genypterus blacodes (Schneider, 1801) (Osteichthyes, Ophidiidae) en el Pacífico Sur Oriental. Biología Pesquera 19: 55-67.

Chong J \& M Aguayo. 1994. Determinación de edad y crecimiento de Seriolella caerulea Guichenot, 1848 de la zona Sur-austral de Chile. Biología Pesquera 23: 59-67.

Chugunova NI. 1963. Age and growth studies in fish. A Systematic Guide for Ichthyologists. Israel Program for Scientific Translations. Izdatel'stvo Akademii Nauk SSSR, Moskva. 1-132. 
Dannevig A. 1933. On the age growth of the cod (Gadus callarias L.) from the Norwegian Skagerrack coast. Report on Norwegian Fishery and Marine Investigation 4(1): 1-145.

Efron B. 1985. Bootstrap confidence imtervals for a class of parametric problems. Biometrika 72(1): 45-48.

Francis RIC. 1990. Back-calculation of fish length: A critical review. Journal of Fish Biology 36: 883-902.

Giussi A. 1996. Descripción del otolito de la merluza de cola (Macruronus magellanicus, Pisces: Merluciidae) y su utilización en la determinación de edad. Instituto Nacional de Investigación y Desarrollo Pesquero. Informe Técnico 10: 1-13.

Jones $\mathbf{R}$ \& $\mathbf{N}$ van Zalinge. 1982. Estimates of mortality rates and populations size for shrimp in Kuwait waters. Kuwait Bulletin of Marine Science 2: 273-288.

Kenchington TJ \& O Augustine. 1987. Age and growth of blue grenadier, Macruronus novaezelandiae (Hector), in Southeastern Australian waters. Australian Journal of Marine and Freshwater Research 38: 625-646.

Ojeda V \& M Aguayo. 1986. Edad y crecimiento de merluza del sur (Merluccius australis) (Gadiformes-Merluciidae). Investigaciones Pesqueras (Chile) 33: 47-59.

Ojeda V, F Cerna, M Aguayo, I Payá \& J Chong. 1998. Estudio de crecimiento y construcción de claves talla edad de merluza de tres aletas y merluza de cola. Informe Final. Fondo de Investigación Pesquera No 97-15: 1-134.

Pantoja V, F Orellana \& E Alarcón. 1973. Evaluación preliminar de los recursos potencialmente explotables situados entre la bahía de Corral e isla Guamblín. Publicaciones Instituto de Fomento Pesquero $N^{\circ}$ 53: 1101.

Pauly D. 1980. On the interrelationships between natural mortality, growth parameters, and mean environmental temperature in 175 fish stock. Journal du Conseil International pour l'Exploration de la Mer 39: 175-192.
Payá I, L Caballero, H Hidalgo \& M Montecinos. 2003. Captura total permisible regionalizada de merluza de cola 2003. Informe final de investigación, Instituto de Fomento Pesquero, $47 \mathrm{pp}$.

Payá I, M Montecinos, J Gonzalez, R Céspedes, L Adasme, $\mathbf{V}$ Ojeda \& S Lillo. 2005. Captura total permisible regionalizada de merluza de cola, 2005. Informe final de investigación. Instituto de Fomento Pesquero, FASE II, 147 pp.

Robson D \& D Chapman. 1961. Catch curves and mortality rates. Transactions of the American Fisheries Society 90 (2): 181-189.

Servicio Nacional de Pesca. 1999. Anuario Estadístico de Pesca 1998. Servicio Nacional de Pesca. Chile, 286 pp.

Servicio Nacional de Pesca. 2001. Anuario Estadístico de Pesca 2000. Servicio Nacional de Pesca. Chile, 194 pp.

Smith DC, GE Fenton, SG Robertson \& SA Short. 1995. Age determination and growth of orange roughy (Hoplostethus atlanticus): a comparison of annulus counts with radiometric ageing. Canadian Journal of Fisheries and Aquatic Sciences 52: 391-401.

Sparre P \& S Venema. 1998. Introducción a la evaluación de recursos pesqueros tropicales. Parte 1- Manual. FAO Documento Técnico de Pesca 306/1. Rev. 2: 1-635.

Taylor CC. 1960. Temperature, growth and mortality, of the Pacific cockle. Journal du Conseil 26: 117-124.

Tomo A \& A Torno. 1987. Determinación de edad y crecimiento en merluza de cola (Macruronus magellanicus Lönnberg). Revista del Museo Argentino de Ciencias Naturales "Bernardino Rivadavia" Hidrobiología 6 (2): 1114.

Young Z, J Chong, H Robotham, P Galvez \& H Gonzalez. 1998. Análisis de la pesquería de merluza de cola en la zona Sur Austral. Informe final. Fondo de Investigación Pesquera, 96-37: 1-97. 